\title{
Autismus: Ein Überblick über Lernvoraussetzungen und Fördermaßnahmen
}

\author{
Jörg Wittwer (i) \\ Albert-Ludwigs-Universität Freiburg, Deutschland
}

\begin{abstract}
Zusammenfassung: Autistische Schülerinnen und Schüler besitzen häufig geringere schulische Kompetenzen als nicht-autistische Schülerinnen und Schüler. Zugleich bestehen große Unterschiede in den schulischen Kompetenzen zwischen autistischen Schülerinnen und Schülern. Nicht selten weisen sie schulische Kompetenzen auf, die niedriger sind, als es aufgrund ihrer kognitiven Fähigkeiten zu erwarten wäre. Um zu verstehen, welche Faktoren das Lernen autistischer Schülerinnen und Schüler beeinflussen, wird in diesem Artikel ein Überblick über die Lernvoraussetzungen autistischer Schülerinnen und Schüler gegeben. Der Überblick zeigt, dass es autistischen Schülerinnen und Schülern schwerfallen kann, Vorwissen abzurufen und in Lernsituationen aktiv anzuwenden, Lernprozesse aufgrund von Einschränkungen in den exekutiven Funktionen angemessen zu steuern, verbale und visuelle Informationen im Arbeitsgedächtnis zu verarbeiten, kognitive und metakognitive Lernstrategien spontan anzuwenden, sich mit Lerninhalten auseinanderzusetzen, die nicht ihren eigenen Interessen entsprechen, und lernabträgliche Emotionen mittels geeigneter Strategien zu regulieren. Um autistische Schülerinnen und Schüler im Lernen zu unterstützen, werden Fördermaßnahmen zu den einzelnen Lernvoraussetzungen vorgestellt. Implikationen für Forschung und Praxis werden diskutiert.
\end{abstract}

Schlüsselwörter: Autismus, Lernvoraussetzungen, Förderung

\section{Autism: An overview of learning prerequisites and interventions}

Abstract: Autistic students often perform academically lower than non-autistic students. At the same time, there is great variability in academic outcomes among autistic students. Not rarely do they have academic outcomes that are discrepant from their general intellectual ability. To understand which factors influence the learning of autistic students, this article provides an overview of the learning prerequisites of autistic students. The overview shows that it can be difficult for autistic students to retrieve prior knowledge and actively use it in learning situations, adequately control learning processes due to impaired executive functions, process verbal and visual information in working memory, spontaneously employ cognitive and metacognitive learning strategies, engage in learning activities that are not in line with their special interests, and regulate emotions by using appropriate strategies. To support the learning of autistic students, interventions for each of the learning prerequisites are presented. Implications for research and practice are discussed.

Keywords: autism, learning prerequisites, interventions

In den letzten Jahrzehnten ist die Zahl autistischer Schülerinnen und Schüler gestiegen (Bachmann, Gerste \& Hoffmann, 2018). Dies gilt auch für die Zahl autistischer Schülerinnen und Schüler, die inklusiv beschult werden (Meindl, Delgado \& Casey, 2020). Die Forschung zeigt, dass es große Unterschiede in den schulischen Kompetenzen zwischen autistischen Schülerinnen und Schülern gibt (z.B. Keen, Webster \& Ridley, 2016). Gleichzeitig besitzen viele autistische Schülerinnen und Schüler geringere schulische Kompetenzen als nicht-autistische Schülerinnen und Schüler (z. B. Bae, Chiang \& Hickson, 2015). Oft sind ihre schulischen Kompetenzen auch niedriger, als es aufgrund ihrer kognitiven Fähigkeiten zu erwarten wäre (z. B. Ashburner, Ziviani \& Rodger, 2010). Zudem verläuft die Entwicklung ihrer schulischen Kompetenzen in vielen
Fällen nicht optimal (z.B. Wei, Christiano, Yu, Wagner \& Spiker, 2015), weshalb das Risiko einer Schulverweigerung erhöht ist (z.B. Munkhaugen, Gjevik, Pripp, Sponheim \& Diseth, 2017).

Mögliche Besonderheiten im Lernen autistischer Schülerinnen und Schüler werden häufig mit autismusspezifischen Merkmalen erklärt (z.B. besonderer Lernstil: Qian \& Lipkin, 2011; Präferenz für Details: Happé \& Frith, 2006). Auch wenn dadurch ein wichtiger Beitrag zum Verständnis des Lernens autistischer Schülerinnen und Schüler geleistet wird, ist die Annahme autismusspezifischer Merkmale gewöhnlich nicht ausreichend, um der Komplexität des Lernens autistischer Schülerinnen und Schüler gerecht zu werden (z.B. Davidson, 2021). Daher wird in diesem Artikel ein transdiagnostischer Ansatz (z.B. 
Holmes et al., 2020) verfolgt und das Lernen autistischer Schülerinnen und Schüler vor dem Hintergrund allgemeiner Lernvoraussetzungen, die für das erfolgreiche Lernen aller Schülerinnen und Schüler wichtig sind, beleuchtet (Hasselhorn \& Gold, 2017). Hierzu wird der aktuelle Forschungstand zu Lernvoraussetzungen autistischer Schülerinnen und Schüler und zu Maßnahmen zur Förderung dieser Lernvoraussetzungen zusammengefasst. Der Überblick ergänzt bisherige Forschungssynthesen, die vor allem schulische Kompetenzen autistischer Schülerinnen und Schüler und deren Förderung in den Bereichen Lesen (z. B. Bailey \& Arciuli, 2020), Schreiben (z.B. Asaro-Saddler, 2016), Mathematik (z. B. King, Lemons \& Davidson, 2016) und Naturwissenschaften (z. B. Barnett, Frankel \& Fisher, 2018) untersuchen.

\section{Autismus}

Autismus ist nach DSM-5 (APA, 2013) eine tiefgreifende Entwicklungsstörung mit Symptomen in sozialer Interaktion (z.B. Schwierigkeiten, Freundschaften aufzubauen), sozialer Kommunikation (z. B. Probleme, bei Gesprächen Blickkontakt zu halten) und Verhalten (z. B. restriktives Interesse an Gegenständen). Das Erscheinungsbild der Symptome kann sehr unterschiedlich sein, weshalb auch von einer Autismus-Spektrum-Störung gesprochen wird. $\mathrm{Au}-$ tismus als Störung zu konzeptualisieren, ist nicht unstrittig. Nach Theunissen (2014) wird durch die Verwendung des Störungsbegriffs Autismus einseitig als ein pathologisches Problem betrachtet, bei dem Defizite im Vordergrund stehen. Daher wird vielfach dafür plädiert, den Begriff der Neurodiversität zu verwenden (Armstrong, 2015). Der Begriff drückt aus, dass Unterschiede in kognitiven Prozessen zwischen Menschen vorteilhafte Aspekte der biologischen Diversität sind (Lambert, Sugita, Yeh, Hunt \& Brophy, 2020). Um die Gefahr einer sprachlichen Stigmatisierung zu reduzieren, wird in diesem Artikel eine identity-first Sprache (d.h. autistische Person) statt einer person-first Sprache (d.h. Person mit Autismus) verwendet (Gernsbacher, 2017).

\section{Lernvoraussetzungen}

Ausgehend von einer kognitivistischen Perspektive auf Lernen als einen Prozess der Informationsverarbeitung haben Hasselhorn und Gold (2017) das Modell der individuellen Voraussetzungen erfolgreichen Lernens (INVOModell) entwickelt. Dieses Modell wird verwendet, um die Lernvoraussetzungen (1) Vorwissen, (2) exekutive Funk- tionen und Arbeitsgedächtnis, (3) Lernstrategien, (4) Selbstkonzept und Motivation sowie (5) Emotionen bei autistischen Schülerinnen und Schülern näher zu beleuchten. Hierzu werden Ergebnisse aus Studien, die autistische Personen mit nicht-autistischen Personen vergleichen, präsentiert. In den Studien sind die Personen gewöhnlich hinsichtlich Alter, Geschlecht und Intelligenz parallelisiert, um zu verhindern, dass beobachtete Unterschiede in den Lernvoraussetzungen zwischen autistischen und nichtautistischen Personen vor allem aufgrund dieser Merkmale auftreten. Die Studien untersuchen hauptsächlich autistische Personen, die mit einem Intelligenzwert von höchstens zwei Standardabweichungen unter dem Mittelwert $(70<$ IQ < 100) keine Intelligenzminderung aufweisen. Hierzu zählen Personen mit hochfunktionalem Autismus und Asperger-Syndrom (zur Problematik der Verwendung dieser Kategorien siehe Alvares et al., 2020, und $\mathrm{zu}$ Unterschieden zwischen beiden Kategorien siehe Chiang, Tsai, Cheung, Brown \& Li, 2014). Dies sind in der

Tabelle 1. Überblick über Effektgrößen aus Metaanalysen zu Unterschieden zwischen autistischen und nicht-autistischen Personen im Hinblick auf Vorwissen, exekutive Funktionen, Arbeitsgedächtnis und Emotionen

\begin{tabular}{|c|c|}
\hline Lernvoraussetzung & Effektgröße \\
\hline \multirow[t]{6}{*}{ Vorwissen } & $\begin{array}{l}\text { Prozedurales Gedächtnis: } \\
g=-0.34^{a^{*}} \text { bis }+0.27^{a^{*}}, g=-0.13^{b^{*}}\end{array}$ \\
\hline & Semantisches Gedächtnis: $g=-0.48^{c}$ \\
\hline & Episodisches Gedächtnis: \\
\hline & Aktiver Abruf: $g=-0.38^{d}$ \\
\hline & Abruf mit Hinweisreizen: $g=-0.08^{d^{*}}$ \\
\hline & Wiedererkennung: $g=-0.15^{d^{*}}$ \\
\hline \multirow{5}{*}{$\begin{array}{l}\text { Exekutive } \\
\text { Funktionen }\end{array}$} & Kognitive Flexibilität: $g=-0.53^{\ominus}, g=-0.57^{f}$ \\
\hline & Flüssigkeit: $g=-0.42^{e}, g=-0.52^{f}$ \\
\hline & Planung: $g=-0.56^{\mathrm{e}}, g=-0.59^{\mathrm{f}}$ \\
\hline & Inhibitionskontrolle: $g=-0.39^{\mathrm{e}}, g=-0.40^{\mathrm{f}}$ \\
\hline & Konzeptbildung: $g=-0.49$ e \\
\hline \multirow[t]{3}{*}{ Arbeitsgedächtnis } & Allgemein: $g=-0.53^{d}, g=-0.54^{e}$ \\
\hline & $\begin{array}{l}\text { Phonologische Schleife: } \\
g=-0.51^{d}, g=-0.56^{f}, d=-0.67^{g}\end{array}$ \\
\hline & $\begin{array}{l}\text { Visuell-räumlicher Notizblock: } \\
g=-0.74^{d}, g=-0.49^{f}, d=-0.73^{g}\end{array}$ \\
\hline \multirow[t]{3}{*}{ Emotionen } & Ängstlichkeit: $d=+0.97^{\mathrm{h}}$ \\
\hline & $\begin{array}{l}\text { Emotionserkennung: } \\
d=-.80^{i} \text { (andere Menschen), } d=-1.16^{j} \text { (selbst) }\end{array}$ \\
\hline & Gefühlsblindheit: $d=+1.51^{k}$ \\
\hline
\end{tabular}

Anmerkungen: $g=$ Hedges $g, d=$ Cohens $d$ (kleiner Effekt: 0.20, mittlerer Ef fekt: 0.50, großer Effekt: 0.80); ${ }^{*}$ Unterschied nicht signifikant; a Foti et al. (2015); ' Obeid et al. (2016); ' Brown et al. (2013); ${ }^{d}$ Desauny et al. (2020);

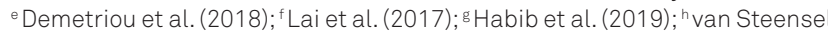
\& Heeman (2017); ' Uljarević \& Hamilton (2013); j Huggins et al. (2021); kKinnaird et al. (2019). 
Regel etwas mehr als die Hälfte aller autistischen Personen (z. B. Postorino et al., 2016). Es ist aber zu beachten, dass bei mehr als einer Standardabweichung unter dem Mittelwert $(70<$ IQ $<85)$ bereits Defizite in der Lernfähigkeit vorhanden sein können (Hasselhorn \& Gold, 2017). Um eine hohe Vergleichbarkeit zwischen autistischen und nicht-autistischen Personen zu erreichen, haben die autistischen Personen in den meisten Studien auch keine anderen Begleitstörungen (z. B. Aufmerksamkeitsstörung), obwohl bei der Mehrheit autistischer Personen mindestens eine Begleitstörung diagnostiziert wird (z. B. Sharma, Gonda \& Tarazi, 2018). Schließlich werden auch Studien mit autistischen Erwachsenen vorgestellt, um zu zeigen, inwieweit Besonderheiten in den Lernvoraussetzungen auch in diesem Altersbereich vorhanden sind. Ein Überblick über Effektgrößen aus Metaanalysen, die autistische Personen mit nicht-autistischen Personen im Hinblick auf Vorwissen, exekutive Funktionen, Arbeitsgedächtnis und Emotionen vergleichen, wird in Tabelle 1 gegeben.

\section{Vorwissen}

Das Vorwissen wird häufig als der wichtigste Faktor für das Lernen betrachtet (Nückles \& Wittwer, 2014). Es beeinflusst nicht nur, welchen Informationen im Lernmaterial Aufmerksamkeit geschenkt wird (z.B. Chi, Feltovich \& Glaser, 1981) und wie zu lernende Informationen miteinander verknüpft werden (z. B. Bransford \& Johnson, 1972), sondern kann auch Schwächen in anderen Lernvoraussetzungen (z.B. geringe Lesekompetenz) kompensieren (z. B. Recht \& Leslie, 1988). Zudem kann fehlerhaftes Vorwissen dazu führen, dass das Erlernen neuer Inhalte beeinträchtigt ist (Prinz, Golke \& Wittwer, 2019). Gedächtnispsychologisch bezieht sich das Vorwissen auf Informationen, die im Langzeitgedächtnis gespeichert sind. Zu dem Langzeitgedächtnis gehören implizites (d.h. perzeptuelles und prozedurales) und explizites (d.h. semantisches und episodisches) Gedächtnis (Tulving, 1995).

\section{Leistung beim direkten Abruf von Gedächtnisinhalten}

In Bezug auf das implizite Gedächtnis zeigen die Metaanalysen von Foti, De Crescenzo, Vivanti, Menghini und Vicari (2015) und Obeid, Brooks, Powers, Gillespie-Lynch und Lum (2016) vergleichbare Leistungen zwischen autistischen und nicht-autistischen Kindern, Jugendlichen und Erwachsenen. Die Metaanalysen berücksichtigen vor allem einfachere Fertigkeiten (z.B. Erlernen von Abfolgen visueller Reize). Daher ist unklar, inwieweit die Ergebnisse auf komplexere Fertigkeiten übertragbar sind. So berichten Patry und Horn (2019) in ihrem Überblick, dass es besonders autistischen Personen schwerfällt, implizites Wissen über Konzepte aufzubauen.
Was das semantische Gedächtnis betrifft, kommen die Überblicksarbeiten von Ben Shalom (2003) und Boucher, Mayes und Bigham (2012) zu dem Ergebnis, dass es generell keine Unterschiede zwischen autistischen und nichtautistischen Personen gibt. Allerdings zeigt die Metaanalyse von Brown, Oram-Cardy und Johnson (2013) zum Textverstehen, dass autistische Personen Beeinträchtigungen im semantischen Gedächtnis aufweisen, wenn es speziell um den Wortschatz geht.

Im Hinblick auf das episodische Gedächtnis weist die Metaanalyse von Desaunay et al. (2020) nach, dass autistische Personen unabhängig vom Alter eine geringere Leistung bei der freien Wiedergabe von Informationen als nicht-autistische Personen erzielen. Wird die Wiedergabe von Informationen durch Hinweisreize unterstützt oder müssen die Informationen lediglich wiedererkannt werden, lassen sich hingegen keine Beeinträchtigungen finden. Offensichtlich sind Einschränkungen in der freien Wiedergabe von Informationen bei autistischen Personen nicht vorrangig mit Schwierigkeiten bei der Enkodierung verbunden, sondern das Ergebnis von Problemen mit dem Abruf (Cooper, Plaisted-Grant, Baron-Cohen \& Simons, 2017). Diese Probleme können damit erklärt werden, dass am Abruf episodischer Inhalte das autonoetische Bewusstsein, das Bewusstsein für das eigene Selbst, beteiligt ist (Desaunay et al., 2020). Dieses Bewusstsein kann als Hinweisreiz genutzt werden, um episodische Inhalte abzurufen. Es wird angenommen, dass autistische Personen ein geringeres Bewusstsein für das eigene Selbst besitzen (Lind, 2010). Daher fällt es ihnen schwerer, sich an episodische Inhalte zu erinnern. Wird die Erinnerung hingegen durch Hinweisreize unterstützt, wird der Zugriff auf die episodischen Inhalte erleichtert. Müssen die episodischen Inhalte lediglich wiedererkannt werden, reicht das Gefühl der Vertrautheit mit den vorgegebenen episodischen Inhalten aus, um die Information zu erinnern. In diesem Fall ist ein Bewusstsein für das eigene Selbst nicht erforderlich (Boucher et al., 2012).

Die Forschung zum episodischen Gedächtnis zeigt auch, dass autistische Personen oft eine geringere Gedächtnisleistung erzielen, wenn die zu lernenden Informationen komplex sind. So fanden Bowler, Gaigg und Gardiner (2014), dass autistische Erwachsene Einzelinformationen genauso gut wie nicht-autistische Erwachsene wiedererkennen konnten. Sollten Kombinationen dieser Einzelinformationen wiedererkannt werden, erbrachten die autistischen Erwachsenen hingegen eine geringere Leistung als die nicht-autistischen Erwachsenen. Auch die Metaanalyse von Desaunay et al. (2020) legt eine Beeinträchtigung der Gedächtnisleistung bei autistischen Personen nahe, wenn die zu erinnernden Informationen semantisch verbunden und somit komplex sind. 


\section{Aktive Nutzung von Gedächtnisinhalten}

Auch wenn keine Wissensunterschiede zwischen autistischen und nicht-autistischen Personen vorliegen sollten, stellt sich die Frage, ob autistische Personen in derselben Weise wie nicht-autistische Personen ihr Wissen aktiv heranziehen, um es in einer konkreten Situation zu nutzen. Channon, Charman, Heap, Crawford und Rios (2001) fanden in ihrer Studie, dass autistische Jugendliche und Erwachsene häufiger als nicht-autistische Jugendliche und Erwachsene an bereits kennengelernte Informationen, die für die Lösung eines Problems relevant waren, erinnert werden mussten. Constable, Ring, Gaigg und Bowler (2018) interessierten sich dafür, nach welchen Prinzipien (z.B. Form, Höhe) autistische und nicht-autistische Erwachsene Holzklötze kategorisierten. Den autistischen Erwachsenen fielen weniger Prinzipien zum Kategorisieren als den nichtautistischen Erwachsenen ein. Król und Król (2019) untersuchten mit Hilfe von Blickbewegungsanalysen, wie autistische und nicht-autistische Jugendliche und Erwachsene auf verfremdeten Fotos Motive (z.B. Blumen) betrachteten, die sie vorher auf Originalfotos gesehen hatten. Nur bei den autistischen Jugendlichen und Erwachsenen war ein besonderes Blickbewegungsmuster zu finden, das auf eine verringerte Nutzung des Vorwissens hinwies. In der Studie von Loth, Gómez und Happé (2011) hörten bzw. lasen Personen im Kindes-, Jugend- und Erwachsenalter Geschichten. Anschließend betrachteten sie Bilder, auf denen Gegenstände mit oder ohne Bezug zu den Geschichten zu sehen waren. In einem Erinnerungstest nannten die autistischen Personen weniger Gegenstände mit Bezug zu den Geschichten als die nicht-autistischen Personen. Wahlberg und Magliano (2004) untersuchten, inwieweit autistische Erwachsene beim Lesen ihr Vorwissen nutzen, um Texte zu interpretieren und mehrdeutige Sachverhalte zu disambiguieren. Es zeigte sich, dass die autistischen Erwachsenen im Vergleich zu den nicht-autistischen Erwachsenen seltener ihr Vorwissen heranzogen, um zu verstehen, was sie lasen.

Dass es autistischen Personen schwerfällt, ihr Vorwissen in einer konkreten Situation anzuwenden, kann unterschiedlich erklärt werden: Nach der Theorie der weak central coherence (Happé \& Frith, 2006) achten autistische Personen besonders stark auf einzelne Informationen und vernachlässigen, dass die einzelnen Informationen gewöhnlich zu einem größeren Ganzen gehören. Daher werden Informationen, die man unter Zuhilfenahme des Vorwissens zusammenfassen könnte (z.B. in Form einer Kategorie), nicht miteinander verknüpft. In ähnlicher Weise besagt die Theorie des enhanced perceptual functioning (Mottron et al., 2013), dass die Informationsverarbeitung autistischer Personen auf den unteren Ebenen (z. B. visuelle Wahrnehmung) besonders stark ausgeprägt ist und TopDown-Prozesse, mit denen Informationen interpretiert wer- den können, einen geringeren Einfluss ausüben. Schließlich nehmen Modelle zum predictive coding (z.B. Van de Cruys et al., 2014) an, dass Personen Vorhersagen über die Umwelt treffen, um adaptiv zu handeln. Dabei treten Vorhersagefehler auf, wenn die Vorhersagen nicht dem sensorischen Input einer Situation entsprechen. Aus den Vorhersagefehlern können Personen lernen, um zukünftig genauere Vorhersagen zu treffen. Es wird vermutet, dass autistische Personen zu einem hohen unflexiblen Vorhersagefehler neigen, aus dem sie keine Regelmäßigkeiten zwischen Situationen ableiten können. Dieser Vorhersagefehler entsteht, wenn aufgrund mangelnder Berücksichtigung von Vorwissen die Vorhersage ungenauer ausfällt, als sie eigentlich sein müsste. Alternativ ist es möglich, dass die Vorhersage zwar vergleichsweise genau ist, aber dennoch ein Vorhersagefehler auftritt, weil unwichtige Details in einer konkreten Situation zu stark berücksichtigt werden.

\section{Exekutive Funktionen und Arbeitsgedächtnis}

Exekutive Funktionen dienen als Regulations- und Kontrollmechanismen dazu, zielorientiert und situationsangepasst zu handeln (Drechsler, 2007). Sie spielen für das Lernen in der Schule eine zentrale Rolle (z.B. Cortés Pascual, Moyano Muñoz \& Quílez Robres, 2019). Demetriou et al. (2018) verglichen in ihrer Metaanalyse die exekutiven Funktionen wie kognitive Flexibilität (z.B. Wechsel zwischen Aufgaben), Flüssigkeit (z.B. Entwicklung von Ideen), Planung (z.B. Verwendung von Strategien), Inhibitionskontrolle (z.B. Unterdrückung von Impulsen) und Bildung von Konzepten (z.B. Anwendung von Regeln) autistischer und nicht-autistischer Personen im Kindes-, Jugend- und Erwachsenenalter. Es zeigte sich bei den autistischen Personen eine geringere Leistung in den exekutiven Funktionen als bei den nicht-autistischen Personen (siehe auch die Metaanalyse von Lai et al., 2017). In Bezug auf kognitive Flexibilität und Flüssigkeit waren die Unterschiede zwischen autistischen und nicht-autistischen Personen im Jugendalter jedoch nicht bedeutsam, was mit einer entwicklungsbedingten, aber temporären Abnahme der exekutiven Funktionen bei nicht-autistischen Personen in diesem Altersbereich erklärt werden kann. Nach der Theorie der exekutiven Dysfunktion (Ozonoff, 1997) haben autistische Personen Schwierigkeiten mit exekutiven Funktionen aufgrund von Besonderheiten im Frontallappen des Gehirns, die den Schwierigkeiten von Personen mit verletzungsbedingten Schäden am Frontallappen des Gehirns ähneln. Wie bei nicht-autistischen Schülerinnen und Schülern sind exekutive Funktionen auch für das Lernen autistischer Schülerinnen und Schüler bedeutsam. St. John, Dawson und Estes (2018) belegten, dass die exekutiven Funktionen autisti- 
scher Kinder im Alter von 6 Jahren deren mathematische Kompetenz im Alter von 9 Jahren vorhersagten.

Das Arbeitsgedächtnis als exekutive Funktion ist der Ort, an dem Informationen bewusst verarbeitet werden. Daher spielt es für das Lernen eine bedeutsame Rolle (z. B. Peng et al., 2018). Nach dem Arbeitsgedächtnismodell von Baddeley (2003) gibt es eine zentrale Exekutive mit zwei Hilfssystemen und einen episodischen Puffer. Die zentrale Exekutive kontrolliert die beiden Hilfssysteme und steuert die Interaktion mit dem Langzeitgedächtnis. Die beiden Hilfssysteme sind die phonologische Schleife, die akustische und verbale Informationen verarbeitet, und der visuell-räumliche Notizblock, der visuelle und räumliche Informationen verarbeitet. Der episodische Puffer speichert temporär episodische Inhalte. Die Kapazität des Arbeitsgedächtnisses ist begrenzt, weshalb nur eine eingeschränkte Zahl an Informationen gleichzeitig verarbeitet werden kann. Die Speicherung von Informationen im Arbeitsgedächtnis geschieht durch eine temporäre Aktivierung von Informationen aus dem Langzeitgedächtnis und durch Wiederholungsprozesse, wodurch die aktivierten Informationen im Fokus der Aufmerksamkeit bleiben (Cowan, 2008).

Habib, Harris, Pollick und Melville (2019) untersuchten in ihrer Metaanalyse das Arbeitsgedächtnis autistischer und nicht-autistischer Personen im Kindes- und Erwachsenenalter. Es zeigte sich bei den autistischen Personen unabhängig vom Alter eine geringere Leistung sowohl für die phonologische Schleife als auch für den visuell-räumlichen Notizblock im Vergleich zu den nicht-autistischen Personen. In ihrer Metaanalyse bestätigten Desaunay et al. (2020) dieses Ergebnis und erklärten es damit, dass aufgrund von Aufmerksamkeitsproblemen bei autistischen Personen neue Informationen im Arbeitsgedächtnis nicht so stark mit korrespondierenden Informationen aus dem Langzeitgedächtnis verknüpft werden und aufgrund von Problemen mit den exekutiven Funktionen nach der Theorie der exekutiven Dysfunktion (Ozonoff, 1997) Prozesse zur Aufrechterhaltung der Informationen beeinträchtigt sind. In der Metaanalyse von Demetriou et al. (2018) ließen sich ebenfalls Einschränkungen im Arbeitsgedächtnis bei autistischen Kindern und Erwachsenen finden. Dass das Arbeitsgedächtnis auch für das Lernen autistischer Schülerinnen und Schüler wichtig ist, bestätigten Oswald et al. (2016), die für autistische und nicht-autistische Jugendliche einen ähnlichen Zusammenhang zwischen Arbeitsgedächtnis und Mathematikkompetenz belegten.

\section{Lernstrategien}

Lernstrategien sind eine zentrale Komponente des selbstregulierten Lernens und beziehen sich auf Aktivitäten, die mit dem Ziel des Lernens durchgeführt werden (Nückles \& Wittwer, 2014). Hierzu gehören kognitive (z. B. Organisieren des Lernstoffs) und metakognitive Aktivitäten (z. B. Überwachen des Lernerfolgs). Viele Studien belegen, dass der Einsatz kognitiver und metakognitiver Lernstrategien das Lernen fördert (z. B. Donker, de Boer, Kostons, Dignath van Ewijk \& van der Werf, 2014).

Wie autistische Personen kognitive Lernstrategien im schulischen Kontext anwenden, wurde bislang kaum untersucht. Allerdings zeigen gedächtnispsychologische Studien, dass autistische Personen selten von sich aus Informationen organisieren, um sie zu lernen (z.B. Minshew \& Goldstein, 2001). Daher fällt es ihnen beispielsweise schwer, neue Konzepte zu bilden (z. B. Patry \& Horn, 2019) oder Probleme zu lösen (z.B. Williams, Mazefsky, Walker, Minshew \& Goldstein, 2014). Diese Schwierigkeiten können auch erklären, warum die Erinnerung an komplexe Informationen, die gewöhnlich organisiert werden müssen, bei autistischen Personen beeinträchtigt ist (Bowler et al., 2014). Vor diesem Hintergrund betrachten Williams, Minshew und Goldstein (2015) Autismus generell als ein Problem der komplexen Informationsverarbeitung. Als Ursache hierfür diskutieren Desaunay et al. (2020) eine neurologisch bedingte Einschränkung in der Fähigkeit, Informationen miteinander zu verknüpfen (relational binding; z. B. Bowler et al., 2014), Beeinträchtigungen in den exekutiven Funktionen (z.B. Maister, Simons \& Plaisted-Grant, 2013) und einen zu geringen Einfluss von Top-DownProzessen (z. B. Loth et al., 2011). Da autistische Personen Informationen häufig nicht spontan organisieren, lernen sie auch gewöhnlich langsamer. In der Studie von Constable et al. (2018) benötigten autistische Erwachsene mehr Versuche als nicht-autistische Erwachsene, um Holzklötze nach Prinzipien zu kategorisieren. Jedoch zeigt die Forschung auch, dass autistische Personen instruiert werden können, Informationen in Beziehung zueinander zu setzen (z. B. Van der Hallen, Evers, Boets, Steyaert, Noens \& Wagemans, 2016). Kennen sie beispielsweise die Regeln, nach denen man Objekte klassifiziert, können sie neues Wissen über Konzepte aufbauen (z.B. Sapey-Triomphe, Sonié, Hénaff, Mattout \& Schmitz, 2018).

In Bezug auf metakognitive Lernstrategien untersuchten Furlano, Kelley, Hall und Wilson (2015), wie genau autistische und nicht-autistische Jugendliche ihre Leistung bei Lese- und Mathematikaufgaben vor und nach der Bearbeitung einschätzten. Leistungsunterschiede in den Leseund Mathematikaufgaben zwischen den autistischen und nicht-autistischen Jugendlichen waren nicht vorhanden. Allerdings überschätzten die autistischen Jugendlichen ihre Leistung sowohl vor als auch nach der Bearbeitung der Lese- und Mathematikaufgaben. Die nicht-autistischen Jugendlichen hingegen waren ziemlich genau in ihren Einschätzungen. Ein ähnliches Ergebnis erhielten 
Furlano und Kelley (2020) in ihrer Studie, an der autistische Kinder und Jugendliche teilnahmen. Die autistischen Kinder und Jugendlichen neigten jedoch nicht mehr zu Überschätzungen, wenn sie nach der Bearbeitung der Aufgaben Rückmeldung zu ihrer Leistung erhielten. Wojcik, Moulin und Souchay (2013) interessierten sich dafür, wie genau autistische Kinder und Jugendliche einschätzten, inwieweit sie Informationen, die sie aktuell nicht erinnerten, zu einem späteren Zeitpunkt wiedererkennen konnten. Es zeigte sich, dass für Informationen aus dem episodischen Gedächtnis (z.B. Lernen von Wortpaaren) autistische Kinder und Jugendliche ungenauere Einschätzungen als nicht-autistische Kinder und Jugendliche vornahmen, während dies für Informationen aus dem semantischen Gedächtnis (z. B. Fragen zum Allgemeinwissen) nicht der Fall war. Die ungenaueren Einschätzungen bei episodischen Informationen können auf das geringere Bewusstsein autistischer Personen für das eigene Selbst zurückgeführt werden (Lind, 2010). Folglich stehen ihnen weniger selbstbezogene Erfahrungen zur Verfügung, um zu entscheiden, ob erlernte, aber aktuell nicht erinnerbare Informationen zu einem späteren Zeitpunkt wiedererkannt werden können. Bei semantischen Informationen treten ungenauere Einschätzungen hingegen nicht auf, weil für ihre Erinnerung keine selbstbezogenen Erfahrungen nötig sind.

Offensichtlich scheint es autistischen Personen schwerzufallen, kognitive und metakognitive Strategien spontan einzusetzen (z. B. Furlano et al., 2015; Minshew \& Goldstein, 2001). Gleichzeitig können kognitive und metakognitive Aktivitäten bei autistischen Personen aber durch Instruktion gefördert werden (z. B. Furlano \& Kelley, 2020; Van der Hallen et al., 2016). Somit fehlen autistischen Personen im Sinne eines Mediationsdefizits (Hasselhorn \& Gold, 2017) nicht grundsätzlich kognitive und metakognitive Strategien. Vielmehr benötigen sie im Sinne eines Produktionsdefizits (Hasselhorn \& Gold, 2017) Hinweise, um kognitive und metakognitive Strategien erfolgreich anzuwenden.

Im Hinblick auf die Art der Informationsverarbeitung wird häufig angenommen, dass autistische Personen einen besonderen Lernstil hätten. Qian und Lipkin (2011) vermuten im Einklang mit Modellen des predictive coding (z.B. Van de Cruys et al., 2014), dass autistische Personen $\mathrm{zu}$ einem Lernstil neigen, der darin besteht, Informationen, die sie in der Auseinandersetzung mit der Umwelt sammeln, einzeln zu speichern. Dieser Lernstil ist effektiv, wenn das Lernmaterial voneinander unabhängige Informationen enthält (z. B. Lernen von Vokabeln). Nicht-autistische Personen tendieren hingegen dazu, nach Regelmäßigkeiten in Informationen zu suchen. Dieser Lernstil ist vorteilhaft, wenn das Lernmaterial Generalisierungen erfordert (z.B. Lernen von Konzepten). Damit überein- stimmend zeigten Sapey-Triomphe et al. (2018), dass autistische Erwachsene im Vergleich zu nicht-autistischen Erwachsenen nicht spontan nach Regelmäßigkeiten im Lernmaterial suchten. Auch Snape, Krott und McCleery (2018) fanden, dass autistische Vorschulkinder bei der Präsentation von zwei Beispielen für ein Konzept, anders als nicht-autistische Vorschulkinder, nicht direkt die Beispiele miteinander verglichen, um Gemeinsamkeiten zu entdecken.

Oft wird auch behauptet, dass autistische Personen einen visuellen Lernstil besäßen (z. B. Rao \& Gagie, 2006). Demnach sollten autistische Personen besonders effektiv lernen, wenn das Lernmaterial visuell gestaltet ist. Trembath, Vivanti, Iacono und Dissanayake (2015) prüften diese Annahme und stellten fest, dass autistische Vorschulkinder im Gegensatz zu nicht-autistischen Vorschulkindern vorgegebene Handlungen mit Gegenständen nicht erfolgreicher ausführten, wenn diese verbal beschriebenen Gegenstände zusätzlich auf Bildern dargestellt wurden. In ähnlicher Weise zeigten Erdődi, Lajiness-O'Neill und Schmitt (2013), dass autistische Kinder und Jugendliche eine geringere Leistung bei Gedächtnisaufgaben als nicht-autistische Kinder und Jugendliche erzielten, wobei diese Leistungsunterschiede bei visuellen Gedächtnisaufgaben größer als bei verbalen Gedächtnisaufgaben waren. Die Ergebnisse deuten darauf hin, dass autistische Personen keinen visuellen Lernstil aufweisen. Visualisierungen können zwar grundsätzlich das Lernen autistischer Personen fördern (z.B. visuelle Helfen; Hume et al., 2021), woraus aber nicht notwendigerweise die Schlussfolgerung zu ziehen ist, dass autistische Personen visuell lernen. Dies deckt sich mit Befunden der lernpsychologischen Forschung, wonach modalitätsspezifische Lernstile generell nicht existieren (Rogowsky, Calhoun \& Tallal, 2015). Offensichtlich können visuelle Informationen sogar die Leistung autistischer Personen verringern. Damit übereinstimmend war in der Metaanalyse von Desaunay et al. (2020) bei autistischen Personen das episodische Gedächtnis für visuelles Material stärker als für verbales Material beeinträchtigt.

\section{Selbstkonzept und Motivation}

Das Selbstkonzept bezieht sich auf die mentale Repräsentation der eigenen Fähigkeiten (Arens, Jansen, Preckel, Schmidt \& Brunner, 2021). Für das Lernen ist vor allem das schulische Selbstkonzept wichtig. Ein hohes schulisches Selbstkonzept kann nicht nur zu einem höheren Lernerfolg (Valentine, DuBois \& Cooper, 2004), sondern ein hoher Lernerfolg auch zu einem höheren schulischen Selbstkonzept führen (Möller \& Marsh, 2013).

Bislang liegen nur wenige Studien zum schulischen Selbstkonzept autistischer Schülerinnen und Schüler vor. 
McCauley et al. (2018) untersuchten das Selbstkonzept in Mathematik und Lesen. Es zeigten sich keine Unterschiede zwischen den autistischen und nicht-autistischen Kindern und Jugendlichen. Allerdings gab es bei den autistischen Kindern und Jugendlichen nur im Bereich der Mathematik einen Zusammenhang zwischen Leistung und Selbstkonzept, während dieser Zusammenhang im Bereich des Lesens nicht zu finden war. Dagegen hingen bei den nichtautistischen Kindern und Jugendlichen Leistung und Selbstkonzept sowohl für Mathematik als auch für Lesen zusammen. Auch wenn offensichtlich keine Beeinträchtigungen im schulischen Selbstkonzept autistischer Schülerinnen und Schüler vorliegen, zeigt die Studie von Ashburner et al. (2010), dass Lehrkräfte autistische Schülerinnen und Schüler als besonders perfektionistisch einschätzten. Solche perfektionistischen Überzeugungen können dysfunktional sein und sich negativ auf das schulische Selbstkonzept auswirken (z. B. Hasselhorn \& Gold, 2017).

Die Motivation bezieht sich allgemein auf die Initiierung, Ausrichtung und Aufrechterhaltung von Aktivitäten, um ein Ziel zu erreichen. Speziell bei der Lernmotivation geht es um das Ziel, Wissen in einem Inhaltsbereich zu erwerben (Schiefele \& Schaffner, 2020). Die zentrale Rolle der Motivation für das Lernen unterstreichen die Ergebnisse der Metaanalyse von Kriegbaum, Becker und Spinath (2018), nach denen die Motivation zusätzlich zur Intelligenz den Lernerfolg vorhersagt. Auch bei autistischen Schülerinnen und Schülern lässt sich ein Zusammenhang zwischen Lernmotivation und schulischen Kompetenzen finden (Keen, Adam \& Simpson, 2021).

Obwohl oft behauptet wird, dass autistische Schülerinnen und Schüler eine besonders geringe Lernmotivation besäßen (z. B. Koegel, Singh \& Koegel, 2010), wurden mögliche Unterschiede in der Lernmotivation zu nicht-autistischen Schülerinnen und Schülern bislang selten untersucht. Chiang et al. (2018) erhoben die Einstellung gegenüber schulischen Aufgaben und fanden, dass autistische Schülerinnen und Schüler eine ungünstigere Einstellung als nichtautistische Schülerinnen und Schüler aufwiesen. Zudem stellten Bae et al. (2015) bei autistischen Kindern eine negativere Einstellung gegenüber Mathematik als bei nichtautistischen Kindern fest. Zajic, Solari, McIntyre, Lerro und Mundy (2020) interessierten sich für die Zeit, die autistische und nicht-autistische Schülerinnen und Schüler mit dem Schreiben einer Erzählung verbrachten. Es zeigte sich, dass die autistischen Schülerinnen und Schüler weniger Zeit für diese Aufgabe als die nicht-autistischen Schülerinnen und Schüler aufwendeten, was zu einer geringeren Qualität ihrer geschriebenen Erzählungen beitrug. Zusätzlich beobachteten Hilvert, Davidson und Gámez (2020), dass es autistischen Schülerinnen und Schülern im Vergleich zu nichtautistischen Schülerinnen und Schülern schwerer fiel, mit dem Schreiben einer Geschichte zu beginnen.
Die Ergebnisse der Studien von Bae et al. (2015) und Chiang et al. (2018) legen nahe, dass autistische Schülerinnen und Schüler dem schulischen Lernen einen vergleichsweise geringen Wert beimessen. Dies kann sich ungünstig auf die Entscheidung, sich mit einem Lerngegenstand überhaupt auseinanderzusetzen, auswirken (Schiefele \& Schaffner, 2020). Zudem scheint es autistischen Schülerinnen und Schülern Probleme zu bereiten, Lernhandlungen zu initiieren (Hilvert et al., 2020) und aufrechtzuerhalten (Zajic et al., 2020). Mögliche Schwierigkeiten mit der Lernmotivation können damit erklärt werden, dass sich autistische Personen vor allem dann mit Gegenständen und Tätigkeiten beschäftigen, wenn diese ihren speziellen Interessen entsprechen (Meindl et al., 2020). Spezielle Interessen (z.B. für Züge) können theoretisch als dispositionale Motivationsmerkmale betrachtet werden, die sich durch eine intensive Auseinandersetzung mit einem Gegenstand, der positive Gefühle auslöst und eine hohe Wichtigkeit aufweist, auszeichnen (Schiefele \& Schaffner, 2020). Grove, Roth und Hoekstra (2016) untersuchten die speziellen Interessen autistischer und nicht-autistischer Erwachsener. Es zeigte sich, dass die autistischen Erwachsenen im Zusammenhang mit ihren speziellen Interessen ein höheres Flowerleben und eine höhere intrinsische Motivation als die nicht-autistischen Erwachsenen berichteten. Die Ursache für das Vorhandensein spezieller Interessen kann theoretisch mit der Monotropismus-Hypothese (Murray, Lesser \& Lawson, 2005) erklärt werden. Demnach können autistische Personen ihre Aufmerksamkeit nur auf wenige Informationen lenken, die Beschäftigung mit diesen Informationen erfolgt dafür aber umso intensiver.

\section{Emotionen}

Emotionen sind psychische Prozesse, die mit einem typischen Erleben (z.B. Freude, Trauer) einhergehen (Frenzel, Götz \& Pekrun, 2020). Emotionen können das Lernen beeinflussen, indem sie sich beispielsweise auf die Auswahl von Lernstrategien oder die Motivation auswirken (Pekrun, 2006). Loderer, Pekrun und Lester (2020) fanden in ihrer Metaanalyse, dass Freude positiv und Ängstlichkeit negativ mit Lernerfolg zusammenhängen. Auch eine angemessene Emotionsregulation, die sich auf eine absichtsvolle Beeinflussung von Emotionen bezieht, ist für das Lernen wichtig (z. B. Graziano, Reavis, Keane \& Calkins, 2007).

Ashburner et al. (2010) untersuchten die Einschätzungen von Lehrkräften zum emotionalen Verhalten autistischer und nicht-autistischer Grundschulkinder. Die Lehrkräfte bewerteten die autistischen Grundschulkinder als ängstlicher, schüchterner, impulsiver, labiler und aggressiver im Vergleich zu den nicht-autistischen Grundschulkin- 
dern. Dies deckt sich mit der Metaanalyse von van Steensel und Heeman (2017), wonach autistische Kinder und Jugendliche eine höhere Ängstlichkeit als nicht-autistische Kinder und Jugendliche aufweisen. Zudem zeigten Cai, Richdale, Uljarević, Dissanayake und Samson (2018) in ihrem Überblick, dass es autistischen Personen altersunabhängig schwerfällt, ihre Emotionen zu regulieren. So fanden Jahromi, Meek und Ober-Reynolds (2012), dass autistische Vorschulkinder im Vergleich zu nicht-autistischen Vorschulkindern bei absichtlich frustrierenden Aufgaben nicht nur stärker und länger resignierten, sondern auch häufiger Vermeidungs- und Ablenkungsstrategien nutzten. Samson, Huber und Gross (2012) beobachteten, dass autistische Erwachsene im Vergleich zu nicht-autistischen Erwachsenen öfter negative Gefühle äußerten, ihre eigenen Gefühle seltener erkannten und auch seltener wirksame Strategien zur Umdeutung emotional belastender Situationen anwendeten. In ähnlicher Weise belegen Metaanalysen, dass autistische Personen Schwierigkeiten haben, die Gefühle anderer Menschen (Uljarević \& Hamilton, 2013) und ihre eigenen Gefühle zu erkennen (Huggins, Donnan, Cameron \& Williams, 2021). Probleme mit der Erkennung und Regulation von Gefühlen können theoretisch mit der Theory of Mind (Rajendran \& Mitchell, 2007) erklärt werden. Demnach fällt es autistischen Personen schwer, die mentalen Zustände anderer Menschen und von sich selbst wahrzunehmen (siehe aber Gernsbacher \& Yergeau, 2019). Man findet bei ihnen auch häufig Alexithymie, eine Persönlichkeitseigenschaft, die sich durch Gefühlsblindheit auszeichnet (Kinnaird, Stewart \& Tchanturia, 2019). Infolgedessen kann es für autistische Personen schwierig sein, auf ihre eigenen Gefühle angemessen zu reagieren.

\section{Förderung}

Der Überblick über die Lernvoraussetzungen autistischer Schülerinnen und Schüler zeigt die Notwendigkeit von Fördermaßnahmen auf: Erstens können autistische Schülerinnen und Schüler zwar grundsätzlich über ein ähnliches Vorwissen wie nicht-autistische Schülerinnen und Schüler verfügen, allerdings können vor allem ihre aktive Erinnerung an episodische Inhalte und ihr semantisches Wissen über Wörter eingeschränkt sein. Dies gilt wahrscheinlich vor allem dann, wenn die zu lernenden Informationen komplex sind. Zweitens kann das Problem bestehen, dass autistische Schülerinnen und Schüler ihr Vorwissen nicht aktiv abrufen, um es in einer Lernsituation zu nutzen. Drittens kann es sein, dass autistische Schülerinnen und Schüler ihren Lernprozess aufgrund von Beeinträchtigungen in den exekutiven Funktionen nicht optimal steuern. Viertens kann die Verarbeitung verbaler und visueller Informationen im Arbeitsgedächtnis erschwert sein. Fünftens kann es passieren, dass autistische Schülerinnen und Schüler kognitive und metakognitive Lernstrategien nicht spontan anwenden. Sie können aber durch entsprechende Hinweise dazu befähigt werden, Lernstrategien zu nutzen. Sechstens ist der Einsatz visueller Lernmaterialien nicht generell vorteilhaft für das Lernen autistischer Schülerinnen und Schüler. Siebtens scheinen autistische Schülerinnen und Schüler ein ähnlich hohes schulisches Selbstkonzept wie nicht-autistische Schülerinnen und Schüler zu besitzen, sie können aber dysfunktionale Überzeugungen aufweisen, die ihr Selbstkonzept gefährden. Achtens kann die Auseinandersetzung mit Lerninhalten, die nicht den eigenen Interessen autistischer Schülerinnen und Schüler entsprechen, reduziert sein. Neuntens können bei autistischen Schülerinnen und Schülern lernabträgliche Emotionen in Verbindung mit einer unzureichenden Emotionsregulation auftreten.

Bei der Gestaltung von Fördermaßnahmen ist zu beachten, dass sich autistische Schülerinnen und Schüler darin unterscheiden können, welche individuellen Stärken und Schwächen sie im Hinblick auf die einzelnen Lernvoraussetzungen aufweisen. Wie ihre Lernvoraussetzungen ausgeprägt sind, kann von unterschiedlichen Faktoren wie der Stärke der autismusspezifischen Symptome (z.B. Van Eylen, Boets, Steyaert, Wagemans \& Noens, 2015), dem Vorhandensein von Begleitstörungen (z.B. Sinzig, Morsch, Bruning, Schmidt \& Lehmkuhl, 2008) und dem Alter (z. B. Desaunay et al., 2020) abhängen.

Die möglichen Beeinträchtigungen in den Lernvoraussetzungen autistischer Schülerinnen und Schüler weisen auf Lernschwierigkeiten hin, die denen von Schülerinnen und Schülern mit einer Lernstörung (z.B. Lese-Rechtschreibstörung) ähneln. Auch Schülerinnen und Schüler mit einer Lernstörung haben oft Schwierigkeiten, ihre Aufmerksamkeit zu lenken, Wissen aufzubauen, Lernstrategien anzuwenden und eine ausreichende Lernmotivation zu entwickeln (z. B. Lauth, Brunstein \& Grünke, 2014). Daher können zur Förderung der Lernvoraussetzungen autistischer Schülerinnen und Schüler grundsätzlich solche Maßnahmen eingesetzt werden, die auch zur Unterstützung von Schülerinnen und Schülern mit einer Lernstörung verwendet werden. Dennoch ist zu beachten, dass sich Lernschwierigkeiten autistischer Schülerinnen und Schüler von denen von Schülerinnen und Schülern mit einer Lernstörung unterscheiden können (Ibrahim, 2020). Erstens kann bei autistischen Personen eine Lernstörung diagnostiziert werden, die unabhängig vom Autismus besteht (z. B. Mayes \& Calhoun, 2006). Zweitens können autistische Personen mit einer Lernstörung stärkere Einschränkungen in ihren Lernvoraussetzungen als autistische Personen ohne Lernstörung aufweisen (z.B. McGuinness 
et al., 2017). Drittens können Lernvoraussetzungen von Personen mit einer Lernstörung beeinträchtigt sein, in denen autistische Personen keine Defizite besitzen (z.B. Clark \& Lum, 2017). Viertens können sich geringe schulische Kompetenzen, die ausschlaggebend für die Diagnose einer Lernstörung sind, in ihrer Erscheinungsform von denen autistischer Schülerinnen und Schüler unterscheiden (z.B. Hendren, Haft, Black, White \& Hoeft, 2018). Beispielsweise sind bei einer Lese-Rechtschreibstörung gewöhnlich Defizite in allen Teilfertigkeiten des Lesens zu finden (Hasselhorn \& Gold, 2017). Autistische Schülerinnen und Schüler hingegen verfügen im Lesen typischerweise über gute Basisfertigkeiten, ihnen fällt jedoch das sinnentnehmende Lesen schwer (Brown et al., 2013). Aufgrund dieser Unterschiede ist die Auswahl konkreter Maßnahmen zur Förderung der Lernvoraussetzungen autistischer Schülerinnen und Schüler stets auf der Grundlage einer umfassenden Individualdiagnostik zu treffen (z.B. Ruble, Dalrymple \& McGrew, 2012). Ein Überblick über mögliche Fördermaßnahmen findet sich in Tabelle 2.

Tabelle 2. Überblick über Maßnahmen zur Förderung der Lernvoraussetzungen autistischer Schülerinnen und Schüler

\begin{tabular}{|c|c|}
\hline Lernvoraussetzung & Fördermaßnahme \\
\hline Vorwissen & $\begin{array}{l}\text { - Direkte Instruktion zur Vermittlung } \\
\text { von Konzepten } \\
\text { - Förderung des Wissensabrufs durch unter- } \\
\text { stütztes Erinnern und Wiedererkennen }\end{array}$ \\
\hline $\begin{array}{l}\text { Exekutive Funktionen } \\
\text { und Arbeitsgedächtnis }\end{array}$ & $\begin{array}{l}\text { - Kognitive Trainings: } \\
\text { - Exekutive Funktionen in Kombination } \\
\text { - Kognitive Flexibilität } \\
\text { - Arbeitsgedächtnis } \\
\text { - Aufmerksamkeit } \\
\text { - Vermeidung lernirrelevanter Informationen }\end{array}$ \\
\hline Lernstrategien & $\begin{array}{l}\text { - Lesestrategien (z.B. QAR-Methode) } \\
\text { - Schreibstrategien (z.B.SRSD-Programm) } \\
\text { - Rechenstrategien (z.B. Schemainstruktion) } \\
\text { - Verhaltensinterventionen: Aufgabenana- } \\
\text { lyse, Modellierung, Prompting, Verstärkung, } \\
\text { Selbstmanagement }\end{array}$ \\
\hline $\begin{array}{l}\text { Selbstkonzept } \\
\text { und Motivation }\end{array}$ & $\begin{array}{l}\text { - Förderung eines Growth Mindset } \\
\text { - Auswählen } \\
\text { - Einstreuung einfacher Aufgaben } \\
\text { - Aktives Antworten } \\
\text { - Berücksichtigung spezieller Interessen }\end{array}$ \\
\hline Emotionen & $\begin{array}{l}\text { - Trainings: } \\
\text { - Emotionserkennung } \\
\text { - Emotionsregulation } \\
\text { - Achtsamkeit }\end{array}$ \\
\hline
\end{tabular}

\section{Vorwissen}

Um den Aufbau von Vorwissen bei autistischen Schülerinnen und Schülern zu fördern, wird häufig die direkte Instruktion als evidenzbasierte Maßnahme verwendet (Hume et al., 2021). Dabei steht gewöhnlich die Vermittlung von Konzepten im Mittelpunkt. Roux, Dion, Barrette, Dupéré und Fuchs (2015) ließen autistische Grundschulkinder unbekannte Wörter aus zu lesenden Texten mit Hilfe von Definitionen, Sätzen, in denen die Wörter eingebettet waren, und Bildern, die die Wörter visualisierten, lernen. Dadurch konnten sich die autistischen Grundschulkinder die Wörter erfolgreich aneignen. Root und Browder (2019) vermittelten autistischen Schülerinnen und Schülern mit Intelligenzminderung mathematische Begriffe, um sie im Lösen mathematischer Textaufgaben zu unterstützen. Wenngleich der Einfluss der Vermittlung mathematischer Begriffe auf den Erwerb mathematischer Fertigkeiten nicht direkt untersucht wurde, weisen andere Studien mit nicht-autistischen Schülerinnen und Schülern solch einen Effekt nach (z.B. Fuchs et al., 2021). Auch Barnett et al. (2018) kommen in ihrem Überblick über evidenzbasierte Fördermaßnahmen in den Naturwissenschaften zu dem Ergebnis, dass die explizite Vermittlung naturwissenschaftlicher Konzepte wichtig ist, um autistischen Schülerinnen und Schülern ein tieferes Verständnis naturwissenschaftlicher Inhalte zu ermöglichen.

Werden neue Informationen gelernt, sind die Informationen als Vorwissen zunächst im episodischen Gedächtnis gespeichert. Um autistische Schülerinnen und Schüler im Abruf gelernter Informationen aus dem episodischen Gedächtnis zu unterstützen, können nach der task-support hypothesis (Bowler et al., 2014) Hinweisreize als Erinnerungsstütze dienen. Auch können Aufgaben eingesetzt werden, die nicht ein aktives Erinnern, sondern lediglich ein Erkennen von Informationen erfordern (Desaunay et al., 2020). Auf diese Weise können Lernmaterialien systematisch an die individuellen Bedürfnisse autistischer Schülerinnen und Schüler angepasst werden (Aguiar, Galy, Godde, Trémaud \& Tardif, 2020).

\section{Exekutive Funktionen und Arbeitsgedächtnis}

Zur Förderung der exekutiven Funktionen autistischer Schülerinnen und Schüler können kognitive Trainings eingesetzt werden. Hierbei werden einzelne exekutive Funktionen durch die wiederholte Durchführung von ähnlichen Aufgaben trainiert. Varanda und Fernandes (2017) führten bei autistischen Kindern und Jugendlichen ein Training zur kognitiven Flexibilität durch. Es zeigte sich, dass das Training effektiv war und auch die Kommunikationsfähig- 
keit der autistischen Kinder und Jugendlichen unterstützte. In der Studie von Kenworthy et al. (2014) nahmen autistische Kinder an einem Training zur Förderung der exekutiven Funktionen in der Schule teil. Das Training verbesserte nicht nur ihre kognitive Flexibilität und ihre Planung, sondern auch ihr Verhalten im Unterricht. Baltruschat et al. (2011) ließen autistische Kinder Arbeitsgedächtnisaufgaben bearbeiten und setzten dabei die Methode der positiven Verstärkung um. Durch das Training steigerten die autistischen Kinder ihre Arbeitsgedächtnisleistung. Zur Förderung der kognitiven Flexibilität und des Arbeitsgedächtnisses setzten de Vries, Prins, Schmand und Geurts (2015) mehrere Trainings ein. Dadurch verbesserten sich die exekutiven Funktionen der autistischen Kinder. Der Effekt war jedoch nicht größer als in einer aktiven Kontrollgruppe autistischer Kinder, die auch die Aufgaben der Trainings erhielten, die Aufgaben jedoch nicht an das individuelle Niveau der autistischen Kinder angepasst waren. Chen, Chang, Marraccini, Cho und Guo (2020) führten ein Aufmerksamkeitstraining durch und stellten fest, dass die autistischen Kinder aufgrund des Trainings ihre kognitive Flexibilität stärker als eine aktive Kontrollgruppe autistischer Kinder steigerten. Spaniol, Shalev, Kossyvaki und Mevorach (2018) untersuchten ebenfalls ein Aufmerksamkeitstraining für autistische Kinder. Das Training verbesserte im Vergleich zu einer aktiven Kontrollgruppe nicht nur die fluide Intelligenz, sondern erhöhte auch die Leistung im Lesen, Schreiben und Rechnen. Macoun, Schneider, Bedir, Sheehan und Sung (2020) verwendeten ein Training zur Förderung der Aufmerksamkeit, der Inhibitionskontrolle und des visuellen Arbeitsgedächtnisses bei autistischen Kindern. Die autistischen Kinder mit Training zeigten größere Verbesserungen in selektiver Aufmerksamkeit, in visuellem Arbeitsgedächtnis, im Rechnen, aber nicht im Lesen als eine passive Kontrollgruppe autistischer Kinder ohne Training.

Dass Trainings die exekutiven Funktionen autistischer Schülerinnen und Schüler fördern können, stimmt mit allgemeinen Befunden aus der Forschung zu kognitiven Trainings überein (z.B. Simons et al., 2016). In zwei Studien (Macoun et al., 2020; Spaniol et al., 2018) ließen sich sogar Transfereffekte auf die schulischen Kompetenzen (z.B. Rechnen) nachweisen. Jedoch ist die Belastbarkeit der Ergebnisse aufgrund der kleinen Stichproben in diesen Studien unklar. Zudem widersprechen die Ergebnisse den Befunden aus Metaanalysen, die generell keine Verbesserungen in schulischen Kompetenzen aufgrund kognitiver Trainings finden (z. B. Melby-Lervåg, Redick \& Hulme, 2016).

Zusätzlich zu kognitiven Trainings können autistische Schülerinnen und Schüler in ihren exekutiven Funktionen auch unterstützt werden, indem sie beim Lernen entlastet werden. Eine solche Entlastung kann sich auf das Arbeitsgedächtnis beziehen. Nach der Cognitive Load Theorie
(Sweller \& Cooper, 1985) kommt es beim Lernen aufgrund der Verarbeitung von Informationen immer zu einer kognitiven Belastung des Arbeitsgedächtnisses. Daher ist es wichtig, Informationen, die für das Lernen nicht unmittelbar relevant sind, zu vermeiden. Die Forschung zeigt, dass es lernförderlich ist, wenn die kognitive Belastung, die durch die Verarbeitung lernirrelevanter Informationen entsteht, reduziert wird (z.B. Vermeidung eines SplitAttention-Effekts; Mayer \& Moreno, 1998). Auch wenn die Cognitive Load Theorie im Zusammenhang mit Autismus diskutiert wird (z.B. Leonard, 2015), haben sich bislang keine empirischen Untersuchungen mit der Wirksamkeit von Lernumgebungen, in denen die lernirrelevante Belastung systematisch verringert wird, für das Lernen autistischer Schülerinnen und Schüler beschäftigt. Indirekte Evidenz für eine erhöhte Wirksamkeit von Lernumgebungen infolge einer Reduzierung lernirrelevanter Informationen stammt von Hanley, Khairat, Taylor, Wilson, Cole-Fletcher und Riby (2017). In ihrer Studie sahen autistische und nicht-autistische Kinder einen videografierten Vortrag einer Lehrkraft. Variiert wurde, ob sich lernirrelevante Informationen im Hintergrund der Lehrkraft befanden. Die autistischen Kinder waren von lernirrelevanten Informationen nicht nur stärker abgelenkt, sondern sie lernten auch weniger als die nicht-autistischen Kinder.

\section{Lernstrategien}

Die Förderung von Lernstrategien bei autistischen Schülerinnen und Schülern wird gewöhnlich im Kontext der Vermittlung von Kompetenzen im Bereich des Lesens, Schreibens oder Rechnens umgesetzt. Beim Lesen stehen oft Strategien zur Verbesserung des Leseverstehens im Mittelpunkt. Beispielsweise wird mit der QAR-Methode (Question-Answer-Relationship) durch explizite Instruktion gelernt, welche Art von Frage (z.B. Frage nach konkreter Information) mit welcher Art von Suche nach Informationen im Text (z. B. bestimmte Wörter in einem Satz) einhergeht (Åsberg \& Dahlgren Sandberg, 2010). Im Hinblick auf die Förderung des Schreibens wird meist das von Harris, Graham und Mason (2006) entwickelte SRSDProgramm (Self-Regulated-Strategy-Development) eingesetzt. In diesem Programm werden domänenspezifische Schreibstrategien zusammen mit metakognitivem Strategiewissen erlernt. In ihrem Überblick kommt Asaro-Saddler (2016) zu dem Ergebnis, dass das Programm dazu geeignet ist, die Qualität des Schreibens und die Überwachungs- und Planungsfertigkeiten auch bei autistischen Schülerinnen und Schülern zu verbessern. Im Bereich des Rechnens wird häufig die Schemainstruktion als eine evidenzbasierte Lernmethode verwendet, um speziell den Erwerb von Fertigkeiten zur Bearbeitung mathematischer 
Textaufgaben zu unterstützen (z.B. Cox \& Root, 2020). Bei der Schemainstruktion wird mit Visualisierungen modelliert, welche Informationen in einer mathematischen Textaufgabe wichtig sind, um das zugrunde liegende mathematische Prinzip zu erkennen, und wie auf dieser Grundlage eine erfolgreiche Mathematisierung umzusetzen ist. Bei autistischen Schülerinnen und Schülern wird die Schemainstruktion häufig modifiziert, indem zusätzlich eine Checkliste verwendet wird, die alle Schritte zur Bearbeitung einer mathematischen Textaufgabe angibt, und Prompts verwendet werden, die dazu auffordern, jeden Schritt durchzuführen.

Um autistische Schülerinnen und Schüler in der Anwendung von Lernstrategien zu unterstützen, haben sich folgende allgemeine Gestaltungsmerkmale von Interventionen als wirksam erwiesen (Hume et al., 2021): Erstens dient eine Aufgabenanalyse dazu, alle Schritte, die autistische Schülerinnen und Schüler zur erfolgreichen Bewältigung einer Aufgabe durchführen müssen, zu identifizieren. Zweitens ist die Durchführung dieser Schritte, etwa von einer Lehrkraft, zu modellieren. Drittens ist es erforderlich, autistische Schülerinnen und Schüler mittels Prompts dazu aufzufordern, die Schritte durchzuführen. Viertens empfiehlt es sich, die richtige Durchführung eines Schritts zu verstärken. Fünftens ist es für das selbstregulierte Lernen nützlich, wenn autistische Schülerinnen und Schüler im Sinne des Selbstmanagement lernen, zwischen angemessenem und unangemessenem Lernverhalten zu unterscheiden.

\section{Selbstkonzept und Motivation}

Auch wenn die Studie von McCauley et al. (2018) keine Beeinträchtigungen im schulischen Selbstkonzept autistischer Schülerinnen und Schüler zeigte, können sich perfektionistische Überzeugungen (z. B. Ashburner et al., 2010) dysfunktional auf das Selbstkonzept auswirken (z. B. Hasselhorn \& Gold, 2017). Eine Möglichkeit, dysfunktionale Überzeugungen zu überwinden, besteht darin, ein growth mindset (Dweck, 2007) zu entwickeln. Nach der Metaanalyse von Sarrasin, Nenciovici, Foisy, Allaire-Duquette, Riopel und Masson (2018) ist diese Methode vor allem bei Schülerinnen und Schülern mit Lernschwierigkeiten wirksam. Lambert et al. (2020) beschreiben in ihrer Einzelfallstudie ausführlich, wie Oscar, ein autistischer Junge, mit Hilfe der Lehrkraft lernte, im Sinne eines growth mindset im Mathematikunterricht Fehler als Lerngelegenheiten zu betrachten: „Usually if you get answers wrong, your brain will grow and grow." (S.503).

Um die Lernmotivation autistischer Schülerinnen und Schüler direkt zu fördern, gibt es nach dem Überblick von Meindl et al. (2020) folgende Methoden: Auswahl, Ein- streuung einfacher Aufgaben und aktives Antworten. Bei der Auswahl geht es darum, so oft wie möglich autistischen Schülerinnen und Schülern die Gelegenheit zu geben, selbst eine Auswahl zu treffen. Die Auswahl kann sich auf die Verwendung eines Stifts, den Sitzplatz (Koegel et al., 2010) oder den zu lernenden Inhalt (Rispoli et al., 2013) beziehen (siehe den Überblick von Reutebuch, El Zein \& Roberts, 2015). Damit die Methode wirksam ist, sollten Gelegenheiten zum Auswählen im Vorhinein geplant und in den Lernprozess systematisch integriert werden (Meindl et al., 2020). Bei der Einstreuung einfacher Aufgaben wird der neue Lernstoff zusammen mit alten Aufgaben, die bereits beherrscht werden, bearbeitet. Die Metaanalyse von Bottini, Vetter, McArdell, Wiseman und Gillis (2018) zeigt, dass unterschiedliche Umsetzungsmöglichkeiten zur Einstreuung einfacher Aufgaben (z. B. hinsichtlich der Art der Aufgaben) eine ähnlich hohe Wirksamkeit aufweisen. Aktives Antworten meint, allen Schülerinnen und Schülern, und somit auch autistischen Schülerinnen und Schülern, möglichst oft die Gelegenheit zu geben, gemeinsam Fragen zu beantworten (Haydon, Marsicano \& Scott, 2013). Dies kann dadurch geschehen, dass Schülerinnen und Schüler auf Fragen, die eine kurze Antwort erfordern, gemeinsam chorisch antworten. Alternativ können Schülerinnen und Schüler Fragen beantworten, indem sie eine Karte mit ihrer Antwort zeigen.

Eine weitere Möglichkeit, die Lernmotivation zu fördern, ist, die speziellen Interessen autistischer Schülerinnen und Schüler zu berücksichtigen. Nach der Metaanalyse von Ninci et al. (2020) kann die Methode vor allem für autistische Personen ohne Intelligenzminderung wirksam sein. Sie wurde bislang jedoch selten zur Förderung des Lernens eingesetzt. Die Studie von El Zein, Solis, Lang und Kim (2014) zeigt, dass ein autistischer Junge mehr Verständnisfragen zu einem Text richtig beantwortete, wenn der Text seine speziellen Interessen berücksichtigte (siehe aber Marshall \& Myers, 2021).

\section{Emotionen}

Um autistische Schülerinnen und Schüler im Erkennen von Emotionen zu unterstützen, werden Trainings eingesetzt. In ihrem Überblick kommen Berggren, FletcherWatson, Milenkovic, Marschik, Bölte und Jonsson (2018) zu dem Ergebnis, dass solche Trainings wirksam sind, aber unklar ist, ob dadurch auch die soziale Interaktion mit anderen Menschen gefördert wird. Zudem gibt es Trainings, in deren Mittelpunkt die Emotionsregulation steht. Conner, White, Beck, Golt, Smith und Mazefsky (2019) fanden, dass ihr Training die von Eltern eingeschätzte Emotionsregulation autistischer Jugendlicher verbesserte und zugleich deren Depressions- und Angstsymptome minderte. 
Ratcliffe, Wong, Dossetor und Hayes (2014) setzten ein Emotionsregulationstraining an Schulen um. Die autistischen Kinder steigerten nach Einschätzung der Lehrkräfte ihre emotionalen Kompetenzen aufgrund des Trainings in der Schule, wohingegen dieser Effekt nach Einschätzung der Eltern zu Hause nicht auftrat. Ein computerbasiertes Training zur Emotionsregulation bei autistischen Kindern haben Kirst et al. (2020) entwickelt. In ihrer Studie zeigte sich, dass das Training nur kurzfristig Emotionserkennung und Empathie förderte, aber längerfristig zu einer verbesserten Emotionsregulation und einer Verringerung autismusspezifischer Symptome führte. Es liegen auch spezielle Achtsamkeitstrainings vor. Ein kombiniertes Training für autistische Jugendliche und deren Eltern entwickelten de Bruin, Blom, Smit, van Steensel und Bögels (2015). Das Training, in dem Selbstkontrollübungen (z.B. Meditation) im Mittelpunkt stehen, führte bei den autistischen Jugendlichen nach eigener Einschätzung zu einer erhöhten Lebensqualität und weniger Grübeln. Die Eltern beobachteten zudem eine Verringerung in den autismusspezifischen Symptomen ihrer Kinder. Salem-Guirgis et al. (2019) fanden in ihrer Studie für dasselbe Training auch eine Verbesserung der Emotionsregulation bei autistischen Jugendlichen.

Offensichtlich können Trainings das Erkennen und die Regulation von Emotionen bei autistischen Schülerinnen und Schülern fördern. Es ist jedoch unklar, inwieweit die Trainings auch das schulische Lernen autistischer Schülerinnen und Schüler unterstützen. Allgemein zeigt die Forschung, dass Fördermaßnahmen zum sozialen und emotionalen Lernen aller Schülerinnen und Schüler sowohl emotionale Kompetenzen als auch schulische Kompetenzen verbessern können (z.B. Durlak, Weissberg, Dymnicki, Taylor \& Schellinger, 2011). Jedoch werden autistische Schülerinnen und Schüler in dieser Forschung bislang nicht berücksichtigt (Daley \& McCarthy, 2020).

\section{Diskussion}

In diesem Artikel wurde auf der Grundlage des INVOModells (Hasselhorn \& Gold, 2017) ein Überblick über den Forschungsstand zu Lernvoraussetzungen autistischer Schülerinnen und Schüler und zu Maßnahmen zur Förderung dieser Lernvoraussetzungen gegeben. Der Überblick berücksichtigt vor allem Studien, die autistische Personen ohne Begleitstörungen untersuchen. Tatsächlich treten Begleitstörungen bei autistischen Personen jedoch häufig auf (Sharma et al., 2018). Daher sollte sich zukünftige Forschung auch verstärkt mit den Lernvoraussetzungen dieser autistischen Schülerinnen und Schüler beschäftigen. Dadurch könnten interessante Erkenntnisse über den Einfluss von Begleitstörungen auf die Lernvoraussetzungen autistischer Schülerinnen und Schüler gewonnen werden (z.B. Sinzig et al., 2008). Diese Erkenntnisse wären auch hilfreich, um Fördermaßnahmen spezifischer auf die individuellen Bedürfnisse autistischer Schülerinnen und Schüler abzustimmen (Ruble et al., 2012).

In dem Überblick wurde die Lesekompetenz autistischer Schülerinnen und Schüler als Lernvoraussetzung nicht berücksichtigt. Sie ist jedoch wichtig, da das sinnentnehmende Lesen bei autistischen Schülerinnen und Schülern oft eingeschränkt ist (Brown et al., 2013). Auch finden sich symptombedingt typischerweise Beeinträchtigungen in der sozialen Kompetenz autistischer Schülerinnen und Schüler, die das Lernen in Gruppen erschweren können (Cheung, Brown, Yu \& Siu, 2021). Vor diesem Hintergrund erscheint es sinnvoll, das INVO-Modell (Hasselhorn \& Gold, 2017) bei autistischen Schülerinnen und Schülern zukünftig um die Lese- und soziale Kompetenz zu ergänzen.

Wie der Überblick verdeutlicht, ist der Umfang an Forschung zu den einzelnen Lernvoraussetzungen autistischer Schülerinnen und Schüler unterschiedlich. Während zu Vorwissen, exekutiven Funktionen, Arbeitsgedächtnis und Emotionen bereits Metaanalysen vorliegen, ist weniger über das Ausmaß möglicher Beeinträchtigungen im Hinblick auf Lernstrategien, Selbstkonzept und Lernmotivation bekannt. Daher wäre weitere Forschung zu diesen Lernvoraussetzungen mit möglichst repräsentativen Stichproben wünschenswert. In diesem Zusammenhang sind auch andere Fragen weitgehend ungeklärt: Erstens wäre es aufschlussreich, Beeinträchtigungen in den Lernvoraussetzungen autistischer Schülerinnen und Schüler längsschnittlich zu analysieren, um Erkenntnisse über ihre zeitliche Stabilität zu erhalten (z. B. Wei et al., 2015). Zweitens könnte der Frage nachgegangen werden, welchen Beitrag die einzelnen Lernvoraussetzungen zum Lernerfolg autistischer Schülerinnen und Schüler leisten (z. B. Keen et al., 2021). Dabei könnte auch untersucht werden, inwieweit sich die einzelnen Lernvoraussetzungen in Abhängigkeit der schulischen Kompetenz (z.B. Lesen, Rechnen) unterschiedlich auf den Lernerfolg auswirken (z.B. Holmes et al., 2020). Drittens wäre die Frage spannend, inwieweit Beeinträchtigungen in einzelnen Lernvoraussetzungen gemeinsame Ursachen aufweisen oder unabhängig voneinander auftreten, sich aber dennoch gegenseitig beeinflussen (Hobson, 2014).

Was die Förderung von Lernvoraussetzungen bei autistischen Schülerinnen und Schülern betrifft, ist anzumerken, dass bisherige Studien oft kleine Stichproben ohne Kontrollgruppe verwenden oder Einzelfälle analysieren. Daher wären mehr randomisierte Kontrollgruppenstudien wünschenswert, um belastbare Erkenntnisse über die Wirksamkeit von Fördermaßnahmen bei autistischen Schülerinnen und Schülern zu erhalten (z. B. Berggren et al., 2018). Dabei sollten auch vermehrt Lernerfolgsmaße eingesetzt 
werden, um den Einfluss der Fördermaßnahmen nicht nur auf die Lernvoraussetzungen, sondern auch auf das Lernen selbst zu untersuchen.

Aus praktischer Sicht kann der Überblick über Lernvoraussetzungen und Fördermaßnahen als Grundlage für die Entwicklung von Trainings für Lehrkräfte dienen (z.B. Bertuccio, Runion, Culler, Moeller \& Hall, 2019). Immer häufiger werden autistische Schülerinnen und Schüler inklusiv beschult, wodurch sich besondere Herausforderungen für Lehrkräfte an Regelschulen ergeben (Meindl et al., 2020). Gleichzeitig besitzen Lehrkräfte oft unzureichende Kenntnisse im Umgang mit autistischen Schülerinnen und Schülern und fühlen sich beim Unterrichten überfordert (z. B. Boujut, Dean, Grouselle \& Cappe, 2016).

Schließlich könnten die in diesem Artikel vorgestellten Erkenntnisse speziell die Pädagogische Psychologie in Deutschland dazu anregen, das Lernen autistischer Schülerinnen und Schüler verstärkt zu untersuchen. Damit würde die Pädagogische Psychologie einen wichtigen Beitrag zu der Erforschung von Inklusion und Umgang mit Heterogenität im Bildungswesen leisten (Richter, Souvignier, Hertel, Heyder \& Kunina-Habenicht, 2019).

\section{Literatur}

Aguiar, Y. P. C., Galy, E., Godde, A., Trémaud, M. \& Tardif, C. (2020). AutismGuide: A usability guidelines to design software solutions for users with autism spectrum disorder. Behaviour \& Information Technology. https://doi.org/10.1080/0144929X.2020. 1856927

Alvares, G. A., Bebbington, K., Cleary, D., Evans, K., Glasson, E. J., Maybery, M. T. et al. (2020). The misnomer of "high functioning autism": Intelligence is an imprecise predictor of functional abilities at diagnosis. Autism, 24, 221 -232. https://doi.org/ 10.1177/1362361319852831

American Psychiatric Association. (2013). Diagnostic and statistical manual of mental disorders. American Psychiatric Association. https://doi.org/10.1176/appi.books.9780890425596

Arens, A. K., Jansen, M., Preckel, F., Schmidt, I. \& Brunner, M. (2021). The structure of academic self-concept: A methodological review and empirical illustration of central models. Review of Educational Research, 91, 34-72. https://doi.org/10.3102/ 0034654320972186

Armstrong, T. (2015). The myth of the normal brain: Embracing neurodiversity. AMA Journal of Ethics, 17, 348 - 352. https://doi. org/10.1001/journalofethics.2015.17.4.msoc1-1504

Asaro-Saddler, K. (2016). Writing instruction and self-regulation for students with autism spectrum disorders. Topics in Language Disorders, 36, 266-283. https://doi.org/10.1097/TLD. 0000000000000093

Åsberg, J. \& Sandberg Dahlgren, A. D. (2010). Discourse comprehension intervention for high-functioning students with autism spectrum disorders: Preliminary findings from a school-based study. Journal of Research in Special Educational Needs, 10, 91 -98. https://doi.org/10.1111/j.1471-3802.2010.01147.x

Ashburner, J., Ziviani, J. \& Rodger, S. (2010). Surviving in the mainstream: Capacity of children with autism spectrum disorders to perform academically and regulate their emotions and behav- ior at school. Research in Autism Spectrum Disorders, 4, 18 - 27. https://doi.org/10.1016/j.rasd.2009.07.002

Bachmann, C. J., Gerste, B. \& Hoffmann, F. (2018). Diagnoses of autism spectrum disorders in Germany: Time trends in administrative prevalence and diagnostic stability. Autism, 22, 283-290. https://doi.org/10.1177/1362361316673977

Baddeley, A. (2003). Working memory: Looking back and looking forward. Nature Reviews Neuroscience, 4, 829 - 839. https://doi. org/10.1038/nrn1201

Bae, Y. S., Chiang, H. M. \& Hickson, L. (2015). Mathematical word problem solving ability of children with autism spectrum disorder and their typically developing peers. Journal of Autism and Developmental Disorders, 45, 2200 - 2208. https://doi.org/10. 1007/s10803-015-2387-8

Bailey, B. \& Arciuli, J. (2020). Reading instruction for children with autism spectrum disorders: A systematic review and quality analysis. Review Journal of Autism and Developmental Disorders, 7,127 - 150. https://doi.org/10.1007/s40489-019-00185-8

Baltruschat, L., Hasselhorn, M., Tarbox, J., Dixon, D. R., Najdowski, A. C., Mullins, R. D. \& Gould, E. R. (2011). Addressing working memory in children with autism through behavioral intervention. Research in Autism Spectrum Disorders, 5, 267 - 276. https:// doi.org/10.1016/j.rasd.2010.04.008

Barnett, J. H., Frankel, A. J. \& Fisher, K. W. (2018). Systematic review of evidence-based interventions in science for students with autism spectrum disorders. Education and Training in Autism and Developmental Disabilities, 53, 128-145.

Ben Shalom, D. (2003). Memory in autism: Review and synthesis. Cortex, 39, 1129-1138. https://doi.org/10.1016/S0010-9452 (08)70881-5

Berggren, S., Fletcher-Watson, S., Milenkovic, N., Marschik, P. B., Bölte, S. \& Jonsson, U. (2018). Emotion recognition training in autism spectrum disorder: A systematic review of challenges related to generalizability. Developmental Neurorehabilitation, 21,141 - 154. https://doi.org/10.1080/17518423.2017.1305004

Bertuccio, R. F., Runion, M. C., Culler, E. D., Moeller, J. D. \& Hall, C. M. (2019). A comparison of autism-specific training outcomes for teachers and paraeducators. Teacher Education and Special Education, 42,338-354. https://doi.org/10.1177/0888406419839771

Bottini, S., Vetter, J., McArdell, L., Wiseman, K. \& Gillis, J. (2018). Task interspersal: A meta-analytic review of effective programming. Review Journal of Autism and Developmental Disorders, 5, 119 -128. https://doi.org/10.1007/s40489-018-0127-7

Boucher, J., Mayes, A. \& Bigham, S. (2012). Memory in autistic spectrum disorder. Psychological Bulletin, 138, 458-496. https://doi. org/10.1037/a0026869

Boujut, E., Dean, A., Grouselle, A. \& Cappe, E. (2016). Comparative study of teachers in regular schools and teachers in specialized schools in France, working with students with an autism spectrum disorder: Stress, social support, coping strategies and burnout. Journal of Autism and Developmental Disorders, 46, 2874-2889. https://doi.org/10.1007/s10803-016-2833-2

Bowler, D. M., Gaigg, S. B. \& Gardiner, J. M. (2014). Binding of multiple features in memory by high-functioning adults with autism spectrum disorder. Journal of Autism and Developmental Disorders, 44, 2355 - 2362. https://doi.org/10.1007/s10803-014-2105-y

Bransford, J. D. \& Johnson, M. K. (1972). Contextual prerequisites for understanding: Some investigations of comprehension and recall. Journal of Verbal Learning and Verbal Behavior, 11, 717 - 726. https://doi.org/10.1016/S0022-5371(72)80006-9

Brown, H. M., Oram-Cardy, J. \& Johnson, A. (2013). A meta-analysis of the reading comprehension skills of individuals on the autism spectrum. Journal of Autism and Developmental Disorders, 43, 932 - 955. https://doi.org/10.1007/s10803-012-1638-1

Cai, R. Y., Richdale, A. L., Uljarević, M., Dissanayake, C. \& Samson, A. C. (2018). Emotion regulation in autism spectrum disorder: Where 
we are and where we need to go. Autism Research, 11, 962 - 978. https://doi.org/10.1002/aur.1968

Channon, S., Charman, T., Heap, J., Crawford, S. \& Rios, P. (2001). Real-life-type problem-solving in Asperger's syndrome. Journal of Autism and Developmental Disorders, 31, 461-469. https:// doi.org/10.1023/A:1012212824307

Chen, M. T., Chang, Y. P., Marraccini, M. E., Cho, M. C. \& Guo, N. W. (2020). Comprehensive attention training system (CATS): A computerized executive-functioning training for school-aged children with autism spectrum disorder. International Journal of Developmental Disabilities [Vorab-Onlinepublikation]. https:// doi.org/10.1080/20473869.2020.1827673

Cheung, P. P. P., Brown, T., Yu, M. \& Siu, A. M. H. (2021). The effectiveness of a school-based social cognitive intervention on the social participation of Chinese children with autism. Journal of Autism and Developmental Disorders, 51, 1894-1908. https:// doi.org/10.1007/s10803-020-04683-1

Chi, M. T. H., Feltovich, P. J. \& Glaser, R. (1981). Categorization and representation of physics problems by experts and novices. Cognitive Science, 5, 121 - 152. https://doi.org/10.1207/s155167 09cog0502_2

Chiang, H. L., Kao, W. C., Chou, M. C., Chou, W. J., Chiu, Y. N., Wu, Y. Y. \& Gau, S. S. (2018). School dysfunction in youth with autistic spectrum disorder in Taiwan: The effect of subtype and ADHD. Autism Research, 11,857-869. https://doi.org/10.1002/aur.1923

Chiang, H. M., Tsai, L. Y., Cheung, Y. K., Brown, A. \& Li, H. (2014). A meta-analysis of differences in IQ profiles between individuals with Asperger's disorder and high-functioning autism. Journal of Autism and Developmental Disorders, 44, 1577-1596. https://doi.org/10.1007/s10803-013-2025-2

Clark, G. M. \& Lum, J. A. G. (2017). Procedural learning in Parkinson's disease, specific language impairment, dyslexia, schizophrenia, developmental coordination disorder, and autism spectrum disorders: A second-order meta-analysis. Brain and Cognition, 117, 41 -48. https://doi.org/10.1016/j.bandc.2017. 07.004

Conner, C. M., White, S. W., Beck, K. B., Golt, J., Smith, I. C. \& Mazefsky, C. A. (2019). Improving emotion regulation ability in autism: The Emotional Awareness and Skills Enhancement (EASE) program. Autism, 23, 1273 -1287. https://doi.org/10. $1177 / 1362361318810709$

Constable, P. A., Ring, M., Gaigg, S. B. \& Bowler, D. M. (2018). Problem-solving styles in autism spectrum disorder and the development of higher cognitive functions. Autism, 22, 597-608. https://doi.org/10.1177/1362361317691044

Cooper, R. A., Plaisted-Grant, K. C., Baron-Cohen, S. \& Simons, J. S. (2017). Eye movements reveal a dissociation between memory encoding and retrieval in adults with autism. Cognition, 159, 127 -138. https://doi.org/10.1016/j.cognition.2016.11.013

Cortés Pascual, A., Moyano Muñoz, N. \& Quílez Robres, A. (2019). The relationship between executive functions and academic performance in primary education: Review and meta-analysis. Frontiers in Psychology, 10, 1582. https://doi.org/10.3389/fpsyg. 2019.01582

Cowan, N. (2008). What are the differences between long-term, short-term, and working memory? Progress in Brain Research, 169, 323 - 338. https://doi.org/10.1016/S0079-6123(07)00020-9

Cox, S. K. \& Root, J. R. (2020). Modified schema-based instruction to develop flexible mathematics problem-solving strategies for students with autism spectrum disorder. Remedial and Special Education, 41, 139-151. https://doi.org/10.1177/0741 932518792660

Daley, S. G. \& McCarthy, M. F. (2020). Students with disabilities in social and emotional learning interventions: A systematic review. Remedial and Special Education [Vorab-Onlinepublikation]. https://doi.org/10.1177/0741932520964917
Davidson, M. M. (2021). Reading comprehension in school-age children with autism spectrum disorder: Examining the many components that may contribute. Language, Speech, and Hearing Services in Schools, 52, 181 -196. https://doi.org/10.1044/ 2020_LSHSS-20-00010

de Bruin, E. I., Blom, R., Smit, F. M., van Steensel, F.J. \& Bögels, S. M. (2015). MYmind: Mindfulness training for youngsters with autism spectrum disorders and their parents. Autism, 19, 906 - 914. https://doi.org/10.1177/1362361314553279

Demetriou, E. A., Lampit, A., Quintana, D. S., Naismith, S. L., Song, Y. J. C., Pye, J. E. et al. (2018). Autism spectrum disorders: A meta-analysis of executive function. Molecular Psychiatry, 23, 1198 -1204. https://doi.org/10.1038/mp.2017.75

Desaunay, P., Briant, A. R., Bowler, D. M., Ring, M., Gérardin, P., Baleyte, J.-M. et al. (2020). Memory in autism spectrum disorder: A meta-analysis of experimental studies. Psychological Bulletin, 146, 377 - 410. https://doi.org/10.1037/bul0000225

de Vries, M., Prins, P. J. M., Schmand, B. A. \& Geurts, H. M. (2015). Working memory and cognitive flexibility-training for children with an autism spectrum disorder: A randomized controlled trial. Journal of Child Psychology and Psychiatry, 56, 566- 576. https://doi.org/10.1111/jcpp.12324

Donker, A. S., de Boer, H., Kostons, D., Dignath van Ewijk, C. C. \& van der Werf, M. P. C. (2014). Effectiveness of learning strategy instruction on academic performance: A meta-analysis. Educational Research Review, 11, 1-26. https://doi.org/10.1016/j. edurev.2013.11.002

Drechsler, R. (2007). Exekutive Funktionen. Zeitschrift für Neuropsychologie, 18, 233 -248. https://doi.org/10.1024/1016-264X. 18.3.233

Durlak, J. A., Weissberg, R. P., Dymnicki, A. B., Taylor, R. D. \& Schellinger, K. B. (2011). The impact of enhancing students' social and emotional learning: A meta-analysis of school-based universal interventions. Child Development, 82, 405 - 432. https://doi.org/ 10.1111/j.1467-8624.2010.01564.x

Dweck, C. S. (2007). Mindset: The new psychology of success. New York: Ballantine Books.

El Zein, F., Solis, M., Lang, R. \& Kim, M. K. (2014). Embedding perseverative interest of a child with autism in text may result in improved reading comprehension: A pilot study. Developmental Neurorehabilitation, 19, 141 -145. https://doi.org/10.3109/175 18423.2014.915893

Erdődi, L., Lajiness-O'Neill, R. \& Schmitt, T. A. (2013). Learning curve analyses in neurodevelopmental disorders: Are children with autism spectrum disorder truly visual learners? Journal of Autism and Developmental Disorders, 43, 880 - 890. https://doi. org/10.1007/s10803-012-1630-9

Foti, F., De Crescenzo, F., Vivanti, G., Menghini, D. \& Vicari, S. (2015). Implicit learning in individuals with autism spectrum disorders: A meta-analysis. Psychological Medicine, 45, 897 -910. https:// doi.org/10.1017/S0033291714001950

Frenzel, A. C., Götz, T. \& Pekrun, R. (2020). Emotionen. In E. Wild \& J. Möller (Hrsg.), Pädagogische Psychologie (S.211-234). Berlin: Springer.

Fuchs, L. S., Seethaler, P. M., Sterba, S. K., Craddock, C., Fuchs, D., Compton, D. L. et al. (2021). Closing the word-problem achievement gap in first grade: Schema-based word-problem intervention with embedded language comprehension instruction. Journal of Educational Psychology, 113, 86-103. https://doi. org/10.1037/edu0000467

Furlano, R. \& Kelley, E. A. (2020). Do children with autism spectrum disorder understand their academic competencies? Journal of Autism and Developmental Disorders, 50, 3101-3113. https:// doi.org/10.1007/s10803-019-03988-0

Furlano, R., Kelley, E. A., Hall, L. \& Wilson, D. E. (2015). Self-perception of competencies in adolescents with autism spectrum dis- 
orders. Autism Research, 8, 761 -770. https://doi.org/10.1002/ aur.1491

Gernsbacher, M. A. (2017). Editorial perspective: The use of person-first language in scholarly writing may accentuate stigma. Journal of Child Psychology and Psychiatry, 58, 859 - 861. https:// doi.org/10.1111/jcpp.12706

Gernsbacher, M. A. \& Yergeau, M. (2019). Empirical failures of the claim that autistic people lack a theory of mind. Archives of Scientific Psychology, 7, 102 - 118. https://doi.org/10.1037/arc0000067

Graziano, P. A., Reavis, R. D., Keane, S. P. \& Calkins, S. D. (2007). The role of emotion regulation in children's early academic success. Journal of School Psychology, 45, 3 - 19. https://doi.org/10.1016/j. jsp.2006.09.002

Grove, R., Roth, I. \& Hoekstra, R. A. (2016). The motivation for special interests in individuals with autism and controls: Development and validation of the Special Interest Motivation Scale. Autism Research, 9,677-688. https://doi.org/10.1002/aur.1560

Habib, A., Harris, L., Pollick, F. \& Melville, C. (2019). A meta-analysis of working memory in individuals with autism spectrum disorders. PLOS ONE, 14, e0216198. https://doi.org/10.1371/journal. pone.0216198

Hanley, M., Khairat, M., Taylor, K., Wilson, R., Cole-Fletcher, R. \& Riby, D. M. (2017). Classroom displays - Attraction or distraction? Evidence of impact on attention and learning from children with and without autism. Developmental Psychology, 53, 1265 - 1275. https://doi.org/10.1037/dev0000271

Happé, F. \& Frith, U. (2006). The weak coherence account: Detailfocused cognitive style in autism spectrum disorders. Journal of Autism and Developmental Disorders, 36, 5-25. https://doi. org/10.1007/s10803-005-0039-0

Harris, K. R., Graham, S. \& Mason, L. H. (2006). Improving the writing, knowledge, and motivation of struggling young writers: Effects of self-regulated strategy development with and without peer support. American Educational Research Journal, 43, 295 - 340. https://doi.org/10.3102/00028312043002295

Hasselhorn, M. \& Gold, A. (2017). Pädagogische Psychologie: Erfolgreiches Lernen und Lehren. Stuttgart: Kohlhammer.

Haydon, T., Marsicano, R. \& Scott, T. M. (2013). A comparison of choral and individual responding: A review of the literature. Preventing School Failure: Alternative Education for Children and Youth, 57, 181 - 188. https://doi.org/10.1080/1045988X.2012.682184

Hendren, R. L., Haft, S. L., Black, J. M., White, N. C. \& Hoeft, F. (2018). Recognizing psychiatric comorbidity with reading disorders. Frontiers in Psychiatry, 9, 101. https://doi.org/10.3389/fpsyt.2018 .00101

Hilvert, E., Davidson, D. \& Gámez, P. B. (2020). Assessment of personal narrative writing in children with and without autism spectrum disorder. Research in Autism Spectrum Disorders, 69, 101453. https://doi.org/10.1016/j.rasd.2019.101453

Hobson, R. P. (2014). The coherence of autism. Autism, 18, 6-16. https://doi.org/10.1177/1362361313497538

Holmes, J., Guy, J., Kievit, R. A., Bryant, A., Mareva, S., Gathercole, S. E. \& CALM Team. (2020). Cognitive dimensions of learning in children with problems in attention, learning, and memory. Journal of Educational Psychology [Vorab-Onlinepublikation]. https://doi.org/10.1037/edu0000644

Huggins, C. F., Donnan, G., Cameron, I. M. \& Williams, J. H. (2021). Emotional self-awareness in autism: A meta-analysis of group differences and developmental effects. Autism, 25, 307-321. https://doi.org/10.1177/1362361320964306

Hume, K., Steinbrenner, J. R., Odom, S. L., Morin, K. L., Nowell, S. W., Tomaszewski, B. et al. (2021). Evidence-based practices for children, youth, and young adults with autism: Third generation review. Journal of Autism and Developmental Disorders [Vorab-Onlinepublikation]. https://doi.org/10.1007/s10803-02004844-2
Ibrahim, I. (2020). Specific learning disorder in children with autism spectrum disorder: Current issues and future implications. Advances in Neurodevelopmental Disorders, 4,103-112. https:// doi.org/10.1007/s41252-019-00141-x

Jahromi, L. B., Meek, S. E. \& Ober-Reynolds, S. (2012). Emotion regulation in the context of frustration in children with high functioning autism and their typical peers. Journal of Child Psychology and Psychiatry, 53,1250 - 1258. https://doi.org/10.1111/ j.1469-7610.2012.02560.x

Keen, D., Adams, D. \& Simpson, K. (2021). Teacher ratings of academic skills and academic enablers of children on the autism spectrum. International Journal of Inclusive Education [VorabOnlinepublikation]. https://doi.org/10.1080/13603116.2021.18 81626

Keen, D., Webster, A. \& Ridley, G. (2016). How well are children with autism spectrum disorder doing academically at school? An overview of the literature. Autism, 20, 276-294. https://doi. org/10.1177/1362361315580962

Kenworthy, L., Anthony, L. G., Naiman, D. Q., Cannon, L., Wills, M. C., Luong-Tran, C. et al. (2014). Randomized controlled effectiveness trial of executive function intervention for children on the autism spectrum. The Journal of Child Psychology and Psychiatry, 55, 374-383. https://doi.org/10.1111/jcpp.12161

King, S. A., Lemons, C. J. \& Davidson, K. A. (2016). Math interventions for students with autism spectrum disorder: A best-evidence synthesis. Exceptional Children, 82, 443 - 462. https:// doi.org/10.1177/0014402915625066

Kinnaird, E., Stewart, C. \& Tchanturia, K. (2019). Investigating alexithymia in autism: A systematic review and meta-analysis. European Psychiatry, 55, 80 - 89. https://doi.org/10.1016/j.eurpsy 2018.09.004

Kirst, S., Diehm, R., Bögl, K., Wilde-Etzold, S., Bach, C., Noterdaeme, M. et al. (2020). Fostering socio-emotional competencies in children with autism using a parent-assisted serious game: A multicenter randomized controlled trial. Manuskript eingereicht zur Publikation. https://doi.org/10.31219/osf.io/pgc7m

Koegel, L. K., Singh, A. K. \& Koegel, R. L. (2010). Improving motivation for academics in children with autism. Journal of Autism and Developmental Disorders, 40, 1057-1066. https://doi.org/ 10.1007/s10803-010-0962-6

Kriegbaum, K., Becker, N. \& Spinath, B. (2018). The relative importance of intelligence and motivation as predictors of school achievement: A meta-analysis. Educational Research Review, 25,120 - 148. https://doi.org/10.1016/j.edurev.2018.10.001

Król, M. \& Król, M. (2019). The world as we know it and the world as it is: Eye-movement patterns reveal decreased use of prior knowledge in individuals with autism. Autism Research, 12, 1386-1398. https://doi.org/10.1002/aur.2133

Lai, C. L. E., Lau, Z., Lui, S. S. Y., Lok, E., Tam, V., Chan, Q. et al. (2017). Meta-analysis of neuropsychological measures of executive functioning in children and adolescents with high-functioning autism spectrum disorder. Autism Research, 10, 911 - 939. https://doi.org/10.1002/aur.1723

Lambert, R., Sugita, T., Yeh, C., Hunt, J. H. \& Brophy, S. (2020). Documenting increased participation of a student with autism in the standards for mathematical practice. Journal of Educational Psychology, 11,494-513. https://doi.org/10.1037/edu 0000425

Lauth, G. W., Brunstein, J. C. \& Grünke, M. (2014). Lernstörungen im Überblick: Arten, Klassifikation, Verbreitung und Erklärungsperspektiven. In G. W. Lauth, M. Grünke \& J. C. Brunstein (Hrsg.), Interventionen bei Lernstörungen: Förderung, Training und Therapie in der Praxis (S.17 - 31). Göttingen: Hogrefe.

Leonard, L. (2015). Cognitive load and Asperger's: Teaching relevance. Journal of Student Engagement: Education Matters, 5, $12-17$. 
Lind, S. E. (2010). Memory and the self in autism: A review and theoretical framework. Autism, 14, 430 - 456. https://doi.org/10 $.1177 / 1362361309358700$

Loderer, K., Pekrun, R. \& Lester, J. C. (2020). Beyond cold technology: A systematic review and meta-analysis on emotions in technology-based learning environments. Learning and Instruction, 70, 101162. https://doi.org/10.1016/j.learninstruc.2018.08.002

Loth, E., Gómez, J. C. \& Happé, F. (2011). Do high-functioning people with autism spectrum disorder spontaneously use event knowledge to selectively attend to and remember context-relevant aspects in scenes? Journal of Autism and Developmental Disorders, 41, 945-961. https://doi.org/10.1007/s10803-010-1124-6

Macoun, S. J., Schneider, I., Bedir, B., Sheehan, J. \& Sung, A. (2020). Pilot study of an attention and executive function cognitive intervention in children with autism spectrum disorders. Journal of Autism and Developmental Disorders [Vorab-Onlinepublikation]. https://doi.org/10.1007/s10803-020-04723-w

Maister, L., Simons, J. S. \& Plaisted-Grant, K. (2013). Executive functions are employed to process episodic and relational memories in children with autism spectrum disorders. Neuropsychology, 27,615-627. https://doi.org/10.1037/a0034492

Marshall, B. \& Myers, C. (2021). Does embedding restricted interests of students with autism in text improve reading comprehension? Developmental Neurorehabilitation, 24, 347-354. https://doi.org/ 10.1080/17518423.2021.1898486

Mayer, R. E. \& Moreno, R. (1998). A split-attention effect in multimedia learning: Evidence for dual processing systems in working memory. Journal of Educational Psychology, 90, 312-320. https://doi.org/10.1037/0022-0663.90.2.312

Mayes, S. D. \& Calhoun, S. L. (2006). Frequency of reading, math, and writing disabilities in children with clinical disorders. Learning and Individual Differences, 16, 145 -157. https://doi.org/10 $.1016 / j$. lindif.2005.07.004

McCauley, J. B., Zajic, M. C., Oswald, T. M., Swain-Lerro, L. E., McIntyre, N. C., Harris, M. A. et al. (2018). Brief report: Investigating relations between self-concept and performance in reading and math for school-aged children and adolescents with autism spectrum disorder. Journal of Autism and Developmental Disorders, 48, 1825-1832. https://doi.org/10.1007/s10803-0173403-y

McGuinness, P. S., Cook, N. E., Bush, H., Lind, H., Vuijk, P. J., Doyle, A. E. et al. (2017). Children with autism spectrum disorder and comorbid specific learning disorder demonstrate cognitive weakness compared to autism spectrum disorder alone. Journal of the American Academy of Child \& Adolescent Psychiatry, 56, S231. https://doi.org/10.1016/j.jaac.2017.09.226

Meindl, J. N., Delgado, D. \& Casey, L. B. (2020). Increasing engagement in students with autism in inclusion classrooms. Children and Youth Services Review, 111, 104854. https://doi.org/10 $.1016 /$ j.childyouth. 2020.104854

Melby-Lervåg, M., Redick, T. S. \& Hulme, C. (2016). Working memory training does not improve performance on measures of intelligence or other measures of "far transfer": Evidence from a metaanalytic review. Perspectives on Psychological Science, 11, 512 - 534. https://doi.org/10.1177/1745691616635612

Minshew, N. J. \& Goldstein, G. (2001). The pattern of intact and impaired memory functions in autism. Journal of Child Psychology and Psychiatry, 42, 1095 -1101. https://doi.org/10.1111/14697610.00808

Möller, J. \& Marsh, H. W. (2013). Dimensional comparison theory. Psychological Review, 120, 544-560. https://doi.org/10.1037/ a0032459

Mottron, L., Bouvet, L., Bonnel, A., Samson, F., Burack, J. A., Dawson, M. et al. (2013). Veridical mapping in the development of exceptional autistic abilities. Neuroscience \& Biobehavioral Reviews, 37, 209 - 228. https://doi.org/10.1016/j.neubiorev.2012.11.016
Munkhaugen, E. K., Gjevik, E., Pripp, A. H., Sponheim, E. \& Diseth, T. H. (2017). School refusal behaviour: Are children and adolescents with autism spectrum disorder at a higher risk? Research in Autism Spectrum Disorders, 41-42, 31-38. https://doi. org/10.1016/j.rasd.2017.07.001

Murray, D., Lesser, M. \& Lawson, W. (2005). Attention, monotropism and the diagnostic criteria for autism. Autism, 9, 139-156. https://doi.org/10.1177/1362361305051398

Ninci, J., Neely, L. C., Burke, M. D., Rispoli, M., Vannest, K.J., Willson, V. et al. (2020). A meta-analysis of single-case research on embedding interests of individuals with autism spectrum disorder. Remedial and Special Education, 41, 296-308. https://doi. org/10.1177/0741932519841692

Nückles, M. \& Wittwer, J. (2014). Lernen und Wissenserwerb. In T. Seidel \& A. Krapp (Hrsg.), Pädagogische Psychologie (S. 225 - 252). Weinheim: Beltz.

Obeid, R., Brooks, P. J., Powers, K. L., Gillespie-Lynch, K. \& Lum, J. A. G. (2016). Statistical learning in specific language impairment and autism spectrum disorder: A meta-analysis. Frontiers in Psychology, 7, 1245. https://doi.org/10.3389/fpsyg.2016.01245

Oswald, T. M., Beck, J. S., losif, A.-M., McCauley, J. B., Gilhooly, L. J., Matter, J. C. et al. (2016). Clinical and cognitive characteristics associated with mathematics problem solving in adolescents with autism spectrum disorder: Factors related to math achievement in ASD. Autism Research, 9, 480 - 490. https://doi. org/10.1002/aur.1524

Ozonoff, S. (1997). Components of executive function in autism and other disorders. In J. Russell (Eds.), Autism as an executive disorder (pp.179-211). Oxford: University Press.

Patry, M. B. \& Horn, E. M. (2019). Schema development in individuals with autism: A review of the literature. Review Journal of Autism and Developmental Disorders, 6, 339-355. https://doi. org/10.1007/s40489-019-00179-6

Pekrun, R. (2006). The control-value theory of achievement emotions: Assumptions, corollaries, and implications for educational research and practice. Educational Psychology Review, 18, 315 - 341. https://doi.org/10.1007/s10648-006-9029-9

Peng, P., Barnes, M., Wang, C., Wang, W., Li, S., Swanson, H. L. et al. (2018). A meta-analysis on the relation between reading and working memory. Psychological Bulletin, 144, 48-76. https:// doi.org/10.1037/bul0000124

Postorino, V., Fatta, L. M., Sanges, V., Giovagnoli, G., De Peppo, L., Vicari, S. et al. (2016). Intellectual disability in autism spectrum disorder: Investigation of prevalence in an Italian sample of children and adolescents. Research in Developmental Disabilities, 48, 193 - 201. https://doi.org/10.1016/j.ridd.2015.10.020

Prinz, A., Golke, S. \& Wittwer, J. (2019). Refutation texts compensate for detrimental effects of misconceptions on comprehension and metacomprehension accuracy and support transfer Journal of Educational Psychology, 111, 957 -981. https://doi. org/10.1037/edu0000329

Qian, N. \& Lipkin, R. M. (2011). A learning-style theory for understanding autistic behaviors. Frontiers in Human Neuroscience, 5, 77. https://doi.org/10.3389/fnhum.2011.00077

Rajendran, G. \& Mitchell, P. (2007). Cognitive theories of autism. Developmental Review, 27, 224-260. https://doi.org/10.1016/j. dr.2007.02.001

Rao, S. M. \& Gagie, B. (2006). Learning through seeing and doing: Visual supports for children with autism. TEACHING Exceptional Children, 38, 26 - 33. https://doi.org/10.1177/004005990603 800604

Ratcliffe, B., Wong, M., Dossetor, D. \& Hayes, S. (2014). Teaching social-emotional skills to school-aged children with autism spectrum disorder: A treatment versus control trial in 41 mainstream schools. Research in Autism Spectrum Disorders, 8, 1722 - 1733. https://doi.org/10.1016/j.rasd.2014.09.010 
Recht, D. R. \& Leslie, L. (1988). Effect of prior knowledge on good and poor readers' memory of text. Journal of Educational Psychology, 80, 16-20. https://doi.org/10.1037/0022-0663 .80.1.16

Reutebuch, C. K., El Zein, F. \& Roberts, G. J. (2015). A systematic review of the effects of choice on academic outcomes for students with autism spectrum disorder. Research in Autism Spectrum Disorders, 20, 1-16. https://doi.org/10.1016/j.rasd.2015 .08 .002

Richter, T., Souvignier, E., Hertel, S., Heyder, A. \& Kunina-Habenicht, O. (2019). Positionspapier zur Lage der Pädagogischen Psychologie in Forschung und Lehre. Psychologische Rundschau, 70, 109 - 118. https://doi.org/10.1026/0033-3042/a000437

Rispoli, M., Lang, R., Neely, L., Camargo, S., Hutchins, N., Davenport, K. et al. (2013). A comparison of within- and across-activity choices for reducing challenging behavior in children with autism spectrum disorders. Journal of Behavioral Education, 22, 66 - 83. https://doi.org/10.1007/s10864-012-9164-y

Rogowsky, B. A., Calhoun, B. M. \& Tallal, P. (2015). Matching learning style to instructional method: Effects on comprehension. Journal of Educational Psychology, 107, 64-78. https://doi. org/10.1037/a0037478

Root, J. R. \& Browder, D. M. (2019). Algebraic problem solving for middle school students with autism and intellectual disability. Exceptionality, 27,118-132. https://doi.org/10.1080/09362835 .2017 .1394304

Roux, C., Dion, E., Barrette, A., Dupéré, V. \& Fuchs, D. (2015). Efficacy of an intervention to enhance reading comprehension of students with high-functioning autism spectrum disorder. Remedial and Special Education, 36, 131-142. https://doi. org/10.1177/0741932514533998

Ruble, L. A., Dalrymple, N. J. \& McGrew, J. H. (2012). Collaborative model for promoting competence and success for students with ASD. Berlin: Springer. https://doi.org/10.1007/978-1-4614-23 32-4

Salem-Guirgis, S., Albaum, C., Tablon, P., Riosa, P. B., Nicholas, D. B., Drmic, I. E. et al. (2019). MYmind: A concurrent group-based mindfulness intervention for youth with autism and their parents. Mindfulness, 10, 1730 - 1743. https://doi.org/10.1007/s12 671-019-01107-9

Samson, A. C., Huber, O. \& Gross, J. J. (2012). Emotion regulation in Asperger's syndrome and high-functioning autism. Emotion, 12, 659-665. https://doi.org/10.1037/a0027975

Sapey-Triomphe, L.-A., Sonié, S., Hénaff, M.-A., Mattout, J. \& Schmitz, C. (2018). Adults with autism tend to undermine the hidden environmental structure: Evidence from a visual associative learning task. Journal of Autism and Developmental Disorders, 48, 3061-3074. https://doi.org/10.1007/s10803-0183574-1

Sarrasin, J. B., Nenciovici, L., Foisy, L.-M. B., Allaire-Duquette, G., Riopel, M. \& Masson, S. (2018). Effects of teaching the concept of neuroplasticity to induce a growth mindset on motivation, achievement, and brain activity: A meta-analysis. Trends in Neuroscience and Education, 12, 22 - 31. https://doi.org/10.1016/ j.tine.2018.07.003

Schiefele, U. \& Schaffner, E. (2020). Motivation. In E. Wild \& J. Möller (Hrsg.), Pädagogische Psychologie (S.163-186). Berlin: Springer.

Sharma, S. R., Gonda, X. \& Tarazi, F. I. (2018). Autism spectrum disorder: Classification, diagnosis and therapy. Pharmacology \& Therapeutics, 190, $91-104$. https://doi.org/10.1016/j.pharm thera.2018.05.007

Simons, D. J., Boot, W. R., Charness, N., Gathercole, S. E., Chabris, C. F., Hambrick, D. Z. et al. (2016). Do "brain-training" programs work? Psychological Science in the Public Interest, 17, 103-186. https://doi.org/10.1177/1529100616661983
Sinzig, J., Morsch, D., Bruning, N., Schmidt, M. H. \& Lehmkuhl, G. (2008). Inhibition, flexibility, working memory and planning in autism spectrum disorders with and without comorbid ADHDsymptoms. Child and Adolescent Psychiatry and Mental Health, 2, 4. https://doi.org/10.1186/1753-2000-2-4

Snape, S., Krott, A. \& McCleery, J. P. (2018). Do children with autism spectrum disorder benefit from structural alignment when constructing categories? Journal of Autism and Developmental Disorders, 48, 2912 - 2924. https://doi.org/10.1007/s10803-0183551-8

Spaniol, M. M., Shalev, L., Kossyvaki, L. \& Mevorach, C. (2018). Attention training in autism as a potential approach to improving academic performance: A school-based pilot study. Journal of Autism and Developmental Disorders, 48, 592-610. https://doi. org/10.1007/s10803-017-3371-2

St. John, T., Dawson, G. \& Estes, A. (2018). Brief report: Executive function as a predictor of academic achievement in school-aged children with ASD. Journal of Autism and Developmental Disorders, 48, 276 - 283. https://doi.org/10.1007/s10803-017-3296-9

Sweller, J. \& Cooper, G. A. (1985). The use of worked examples as a substitute for problem solving in learning algebra. Cognition and Instruction, 2, 59 - 89. https://doi.org/10.1207/s1532690xci 0201_3

Theunissen, G. (2014). Menschen im Autismus-Spektrum: Verstehen - annehmen - unterstützen. Stuttgart: Kohlhammer.

Trembath, D., Vivanti, G., lacono, T. \& Dissanayake, C. (2015). Accurate or assumed:Visual learning in children with ASD. Journal of Autism and Developmental Disorders, 45, 3276-3287. https:// doi.org/10.1007/s10803-015-2488-4

Tulving, E. (1995). Organization of memory: Quo vadis? In M. S. Gazzaniga (Eds.), The cognitive neurosciences (pp.839-853). The MIT Press.

Uljarević, M. \& Hamilton, A. (2013). Recognition of emotions in autism: A formal meta-analysis. Journal of Autism and Developmental Disorders, 43, 1517 -1526. https://doi.org/10.1007/s10 803-012-1695-5

Valentine, J. C., DuBois, D. L. \& Cooper, H. (2004). The relation between self-beliefs and academic achievement: A meta-analytic review. Educational Psychologist, 39, 111 - 133. https://doi.org/10 .1207/s15326985ep3902_3

Van de Cruys, S., Evers, K., Van der Hallen, R., Van Eylen, L., Boets, B., de-Wit, L. et al. (2014). Precise minds in uncertain worlds: Predictive coding in autism. Psychological Review, 121, 649-675. https://doi.org/10.1037/a0037665

Van der Hallen, R., Evers, K., Boets, B., Steyaert, J., Noens, I. \& Wagemans, J. (2016). Visual search in ASD: Instructed versus spontaneous local and global processing. Journal of Autism and Developmental Disorders, 46, 3023-3036. https://doi.org/10 .1007/s10803-016-2826-1

Van Eylen, L., Boets, B., Steyaert, J., Wagemans, J. \& Noens, I. (2015). Executive functioning in autism spectrum disorders: Influence of task and sample characteristics and relation to symptom severity. European Child \& Adolescent Psychiatry, 24, 1399-1417. https://doi.org/10.1007/s00787-015-0689-1

van Steensel, F. \& Heeman, E. J. (2017). Anxiety levels in children with autism spectrum disorder: A meta-analysis. Journal of Child and Family Studies, 26, $1753-1767$. https://doi.org/10 .1007/s10826-017-0687-7

Varanda, C. \& Fernandes, F. (2017). Cognitive flexibility training intervention among children with autism: A longitudinal study. Psicologia: Reflexão e Crítica, 30, 15. https://doi.org/10.1186/ s41155-017-0069-5

Wahlberg, T. \& Magliano, J. P. (2004). The ability of high function individuals with autism to comprehend written discourse. Discourse Processes, 38, 119-144. https://doi.org/10.1207/s1532 6950dp3801_5 
Wei, X., Christiano, E. R., Yu, J. W., Wagner, M. \& Spiker, D. (2015). Reading and math achievement profiles and longitudinal growth trajectories of children with an autism spectrum disorder. Autism, 19, 200 - 210. https://doi.org/10.1177/136236131 3516549

Williams, D. L., Mazefsky, C. A., Walker, J. D., Minshew, N. J. \& Goldstein, G. (2014). Associations between conceptual reasoning, problem solving, and adaptive ability in high-functioning autism. Journal of Autism and Developmental Disorders, 44, 2908-2920. https://doi.org/10.1007/s10803-014-2190-y

Williams, D. L., Minshew, N. J. \& Goldstein, G. (2015). Further understanding of complex information processing in verbal adolescents and adults with autism spectrum disorders. Autism, 19,859 - 867. https://doi.org/10.1177/1362361315586171

Wojcik, D. Z., Moulin, C. J. A. \& Souchay, C. (2013). Metamemory in children with autism: Exploring "feeling-of-knowing" in episodic and semantic memory. Neuropsychology, 27, 19-27. https://doi. org/10.1037/a0030526

Zajic, M. C., Solari, E. J., Mclntyre, N. S., Lerro, L. \& Mundy, P. C. (2020). Task engagement during narrative writing in school-age children with autism spectrum disorder compared to peers with and without attentional difficulties. Research in Autism Spectrum Disorders, 76, 101590. https://doi.org/10.1016/j.rasd .2020 .101590

\section{Historie}

Manuskript eingereicht: 02.02.2021

Manuskript nach Revision angenommen: 06.07.2021

Onlineveröffentlichung: 30.07.2021

\section{Danksagung}

Ich danke Lena Wimmer für ihre hilfreichen Anmerkungen zu einer vorläufigen Fassung dieses Artikels.

\section{Förderung}

Open-Access-Veröffentlichung ermöglicht durch die Albert-Ludwigs-Universität Freiburg.

\section{ORCID}

Jörg Wittwer

(D) https://orcid.org/0000-0001-9984-7479

\section{Prof. Dr. Jörg Wittwer}

Universität Freiburg

Institut für Erziehungswissenschaft

Remparstraße 11

79098 Freiburg

Deutschland

joerg.wittwer@ezw.uni-freiburg.de 